\title{
Time to Pay Attention: Attentional Performance Time-Stamped Prefrontal Cholinergic Activation, Diurnality, and Performance
}

\author{
Giovanna Paolone, Theresa M. Lee, and Martin Sarter \\ Department of Psychology, University of Michigan, Ann Arbor, Michigan 48109
}

\begin{abstract}
Although the impairments in cognitive performance that result from shifting or disrupting daily rhythms have been demonstrated, the neuronal mechanisms that optimize fixed-time daily performance are poorly understood. We previously demonstrated that daily practice of a sustained attention task (SAT) evokes a diurnal activity pattern in rats. Here, we report that SAT practice at a fixed time produced practice time-stamped increases in prefrontal cholinergic neurotransmission that persisted after SAT practice was terminated and in a different environment. SAT time-stamped cholinergic activation occurred regardless of whether the SAT was practiced during the light or dark phase or in constant-light conditions. In contrast, prior daily practice of an operant schedule of reinforcement, albeit generating more rewards and lever presses per session than the SAT, neither activated the cholinergic system nor affected the animals' nocturnal activity pattern. Likewise, food-restricted animals exhibited strong food anticipatory activity (FAA) and attenuated activity during the dark phase but FAA was not associated with increases in prefrontal cholinergic activity. Removal of cholinergic neurons impaired SAT performance and facilitated the reemergence of nocturnality. Shifting SAT practice away from a fixed time resulted in significantly lower performance. In conclusion, these experiments demonstrated that fixed-time, daily practice of a task assessing attention generates a precisely practice time-stamped activation of the cortical cholinergic input system. Time-stamped cholinergic activation benefits fixedtime performance and, if practiced during the light phase, contributes to a diurnal activity pattern.
\end{abstract}

\section{Introduction}

Animals schedule daily routines and tasks with great precision, assisted in part by photic and nonphotic zeitgebers. Circadian shifts affect the timing of behavior and impair performance, particularly of tasks involving cognitive operations (Stroebel, 1967; Holloway and Wansley, 1973; Fekete et al., 1985; Daan, 2000; Schmidt et al., 2007; Gerstner and Yin, 2010). Moreover, the performance of vulnerable populations and patients with neuropsychiatric and degenerative disorders is extremely sensitive to phase shifts and circadian dysregulation (Meck, 1991; Lustig and Meck, 2001; Bergua et al., 2006; Reid et al., 2011; Wulff and Joyce, 2011). However, the neuronal mechanisms that optimize daily, time-fixed performance have remained largely undefined (but see Chee et al., 2006).

We previously reported that daily practice of a sustained attention task (SAT), but not of an operant schedule of reinforcement, daily practice of a maze task, daily handling, or restricted

Received May 10, 2012; revised June 20, 2012; accepted July 10, 2012.

Author contributions: G.P., T.M.L., and M.S. designed research; G.P. performed research; G.P. analyzed data; G.P., T.M.L., and M.S. wrote the paper.

This work was supported by United States Public Health Service Grant MH079084 (T.M.L., M.S.). We thank Damon A. Young (now at Pfizer) and Danielle Lamy for technical assistance. We also thank Fabiana Magnosi for generating illustrations.

Correspondence should be addressed to Martin Sarter, Department of Psychology, University of Michigan, 530 Church Street, 4032 East Hall, Ann Arbor, MI 48109-8862. E-mail: msarter@umich.edu.

T. M. Lee's present address: University of Tennessee, 312 Ayres Hall, 1403 Circle Drive, Knoxville, TN 37996-1330. DOI:10.1523/JNEUROSCI.2271-12.2012

Copyright $\odot 2012$ the authors $\quad 0270-6474 / 12 / 3212115-14 \$ 15.00 / 0$ water access, elicits a diurnal activity pattern in rats (Gritton et al., 2009, 2012). Our evidence indicates close interactions between the neuronal mechanisms mediating SAT performance and circadian pacemakers, rendering attentional performance to be particularly vulnerable to circadian abnormalities. Human studies have confirmed that attentional processes are potently influenced by circadian variables (Schmidt et al., 2007; Matchock and Mordkoff, 2009; Mollicone et al., 2010; van der Heijden et al., 2010).

Sustained attention performance depends on the integrity of the cortical cholinergic input system (McGaughy et al., 1996; McGaughy and Sarter, 1998). Two distinct modes of cholinergic neurotransmission mediate such performance (Hasselmo and Sarter, 2011). First, attentional cue-evoked, brief (seconds) increases in cholinergic neurotransmission are generated via local cortical circuitry, requiring cue-evoked thalamic input (Parikh et al., 2007, 2008). These brief "transients" are hypothesized to facilitate the cognitive processes involved in cue detection [as defined by Posner et al. (1980)]. Second, a more tonically active cholinergic input system (changes over tens of seconds to minutes) modulates these cue-evoked glutamatergic-cholinergic interactions (Parikh et al., 2010). Importantly, measures of tonic cholinergic activity, using microdialysis, are not confounded by transients that can be recorded amperometrically with enzymecoated microelectrodes (Sarter and Parikh, 2005; Paolone et al., 2010). Tonic levels of cholinergic activity are elevated during SAT performance (Dalley et al., 2001; Arnold et al., 2002) and further increase in response to demands on the cognitive control of attention (St. Peters et al., 2011a). 
The present experiments originally aimed at determining the consequences of SAT performance-induced diurnality on the circadian regulation of tonic cholinergic activity (Hut and Van der Zee, 2011). The discovery of increases in cholinergic neurotransmission that are precisely synchronized to the time of prior SAT performance motivated a series of experiments that collectively demonstrate that time-stamped cholinergic activity is a specific consequence of fixed-time SAT practice in the light or dark phase or under constant-light conditions, it contributes to the diurnality that results from SAT practice during the light phase, and it benefits daily, fixed-time attentional performance.

\section{Materials and Methods}

Subjects. Adult male Sprague Dawley rats (Charles River Laboratories), aged 3-5 months and weighing between 250 and $300 \mathrm{~g}$ at the beginning of the experiments, were used. Animals were individually housed in a temperature- $\left(23^{\circ} \mathrm{C}\right)$ and humidity-controlled $(45 \%)$ environment under a $12 \mathrm{~h}$ light/dark (12:12 LD) schedule unless otherwise reported. Animals were handled extensively before the beginning of task training. Animals were water deprived by limiting access to a 15 min period following each behavioral (operant) training session. Water was also provided as a reward for correct responses during task performance (see below). On days not tested, the duration of water access was increased to 30 min. Food (Rodent Chow; Harlan Teklad), unless noted otherwise, was available ad libitum. Body weights were recorded weekly. All procedures were conducted in adherence with protocols approved by the University Committee on Use and Care of Animals at the University of Michigan and in laboratories accredited by the Association for Assessment and Accreditation of Laboratory Animal Care. The number of animals per group of condition is reported in Results by indicating the degrees of freedom for all major analyses.

Monitoring of circadian activity patterns. Animals were randomly assigned to one of the three behavioral training/practice times, beginning $4 \mathrm{~h}$ into the light phase [zeitgeber time 4 (ZT4)] or dark phase (ZT16) or constant light (LL) (Gritton et al., 2009, 2012). We used motion detectors (placed $25 \mathrm{~cm}$ above the home cages; 20 detectors; Slimline PIR; SmartHome) and running wheels (25 wheels; Mini Mitter) to monitor daily activity patterns. Motion detectors were demonstrated to effectively and validly measure entrained activity rhythms (Mistlberger et al., 2009). Activity counts were collected in $10 \mathrm{~min}$ bins via VitalView software (Mini Mitter; version 4.0) and analyzed using ActiView software (Mini Mitter; version 1.0). Although running wheel activity has often been considered to represent merely an alternative method for recording general activity and response comparably to major entrainment stimuli such as food or light (Campbell and Lynch, 1968; Rosenwasser et al., 1996), the behavioral and environmental variables governing this behavior remain not well understood (Collier and Hirsch, 1971; Sherwin, 1998). Important manipulations involved in the current experiments, particularly food deprivation, may differentially affect wheel running and cage activity patterns (Treichler and Hall, 1962), reflecting the complex interactions between extrinsic motivational and intrinsic wheel running-associated incentive processes (Atalayer and Rowland, 2011). Therefore, both methods were used, allowing us to address potential method-based biases. As was the case in our prior experiments using both methods (Gritton et al., 2009), SAT practice at ZT4 evoked a persistent diurnal activity pattern measured by both motion detectors (see Fig. $2 a$ ) and running wheels (see Fig. $5 b$ ).

We determined the circadian activity patterns of animals exhibiting criterion performance in the SAT or behavioral control procedures. Activity ratios (LD ratio) were calculated by dividing the sum of the activity counts during the light phase by the sum of the counts during the dark phase of each $24 \mathrm{~h}$ cycle. LD ratios $>1$ indicate a diurnal activity preference. More restricted analyses focused on the activity pattern during periods of prior task performance. For these analyses, activity counts were collected over a $3 \mathrm{~h}$ interval that began $90 \mathrm{~min}$ before (the previous) task onset. Counts from these intervals were compared with counts collected during the corresponding $3 \mathrm{~h}$ period of the subsequent $12 \mathrm{~h}$ phase [light, dark, or constant light (LL), depending on group].
Behavioral apparatus, SAT task acquisition, and performance criteria. Behavioral training took place either during the light phase, beginning $4 \mathrm{~h}$ into the light phase (ZT4) or into the dark phase (ZT16), or in constant-light condition (LL). Except for LL animals, SAT training began after a 1 week acclimation period during which animals were handled twice at randomly selected times, and food and water were available ad libitum. LL animals began SAT training 3 weeks after they were moved from LD to LL and when they exhibited free running activity patterns (see Results).

Behavioral training and testing took place using 12 operant chambers located inside sound-attenuating cubicles (MED Associates). The chambers were equipped with a water dispenser $(40-45 \mu l$ of water per delivery). Although even white light, when presented during the rats' inactive period, does not entrain their activity (Daan and Pittendrigh, 1976), the chambers' cue lights, centered on the intelligence panel, and houselights, located on rear wall, were replaced by red LEDs (586 series; peak wavelength, $630 \mathrm{~nm}$; Dialight Corporation). Before the sessions involving the collection of dialysates from prefrontal cortex (below), animals were moved to modified operant chambers and trained again to criterion performance. These chambers featured a taller recessed water delivery area $(9.0 \times 5.0 \mathrm{~cm}$, height by width) to allow animals to drink after cannulation and, subsequently, inlet and outlet lines connected to the microdialysis probe. Signal presentation, lever operation, reinforcement delivery, and data collection were controlled by a Pentium PC and Med-PC for Windows software (version 4.1.3; MED Associates).

The SAT task and evidence in support of the validity of performance measures in terms of indicating sustained attention performance, in mice, rats, and humans, were described earlier (McGaughy and Sarter, 1995; Demeter et al., 2008; St. Peters et al., 2011b). Animals were initially trained to lever press in accordance with a modified fixed ratio-1 (FR-1) schedule for water reinforcement. Following at least three consecutive sessions per day with $>120$ reinforced lever presses each, animals entered the first stage of SAT training during which they were trained to discriminate between a signal ( $1 \mathrm{~s}$ illumination of the central panel light) and a nonsignal (no illumination) event. Two seconds following such events, the levers were extended into the chamber. On signal trials, a response on the left lever was reinforced and termed a "hit," while a response on the right lever was not reinforced and termed a "miss." On nonsignal trials, a response on the right lever was reinforced and termed a "correct rejection," while a response on the left lever was not reinforced and termed a "false alarm." One-half of the animals were trained using the reverse set of rules. If no response occurred within $4 \mathrm{~s}$, the levers were retracted and an omission was recorded. Signal and nonsignal events were presented in pseudorandom order for 81 trials each (total of 162 trials) per session. The intertrial interval (ITI) was $12 \pm 3 \mathrm{~s}$. During this stage of training, incorrect responses were followed by up to three correction trials in which the previous trial (signal or nonsignal) was repeated. If an incorrect response occurred again on the third correction trial, a forced trial was initiated in which only the correct lever was extended for $90 \mathrm{~s}$ or until the animal made a response. When the forced trial was a signal trial, the central panel light remained illuminated while the left lever was extended. The house light was not illuminated during this training stage. Following at least 5 consecutive days of stable performance, defined as $>70 \%$ hits and $>70 \%$ correct rejections, multiple signal durations (500, $50,25 \mathrm{~ms}$ ) were introduced. Trial type and signal duration continued to be pseudorandomly determined for each trial. Session length was set at $40 \mathrm{~min}$ to allow for post hoc analysis of performance over five blocks ( 8 min per block). The pseudorandom selection of trial type (signal vs nonsignal) and signal duration was designed to ensure that approximately one-half of the trials per block were signal trials, and that equivalent numbers of 500, 50, and $25 \mathrm{~ms}$ signals were presented during each block. At this point, correction trials and forced trials were discontinued, and the event rate was increased by reducing the ITI to $9 \pm 3 \mathrm{~s}$. Following at least $7 \mathrm{~d}$ of stable performance, defined by at least $70 \%$ hits to $500 \mathrm{~ms}$ signals, $70 \%$ correct rejections, and $<20 \%$ omissions, animals began training in the final version of the task. The final version was identical with the previous training stage except that the house light was illuminated throughout the session. This key final modification requires the animals to constrain their behavior and, presumably, to maintain persis- 
tent attention to the intelligence panel to monitor the signal source. On average, animals required 12 weeks of daily practice to reach criterion performance. Criterion performance at this stage was defined as $>70 \%$ hits to $500 \mathrm{~ms}$ signals, $>70 \%$ correct rejections, and $<20 \%$ omissions for five consecutive sessions.

After attaining criterion performance, animals underwent surgery for implantation of a unilateral guide cannula in the medial prefrontal cortex (mPFC) (below). After animals recovered from surgery, they resumed their water deprivation and behavioral training. After again attaining stable performance in the final stage of the sustained attention task for at least $5 \mathrm{~d}$, animals were transferred to the dialysis chambers $(35.0 \mathrm{~cm}$ height, $38.0 \mathrm{~cm}$ diameter) lined with corncob bedding. A separate group of rats was left undisturbed until their activity patterns reversed from the SAT performance-associated diurnal activity pattern to their regular nocturnal pattern (henceforth termed "renocturnal"). They were then returned to the dialysis chambers and left undisturbed for $3 \mathrm{~d}$ under mock-microdialysis conditions (defined below; see also Fig. 1a).

Measures of SAT performance. For each session, hits, misses, correct rejections, false alarms, and omissions were recorded. The relative number of hits (hits/hits + misses) was calculated for each signal length, and the relative number of correct rejections (correct rejections/correct rejections + false alarms) was also calculated. As an overall measure of attentional performance that integrates both the relative number of hits $(h)$ and the relative number of false alarms $(f)$, an overall performance score (SAT score) was calculated in accordance to the following: SAT $=$ $(h-f) /[2(h+f)-(h+f) 2]$. This index was derived from the sensitivity index (Frey and Colliver, 1973), except that the SAT score is based on the relative number of hits and false alarms, as opposed to the probabilities for hits and false alarms, and thus is not confounded by errors of omission. SAT scores range from +1.0 to -1.0 , with +1.0 indicating that all responses were hits and correct rejections, 0 indicating an inability to discriminate between signal and nonsignal events, and -1.0 indicating that all responses were misses and false alarms. SAT scores were calculated for each signal duration $\left(\mathrm{SAT}_{500,50,25}\right)$ or averaged over durations. Errors of omission were recorded separately. Performance measures were calculated for each of the five task blocks.

Fixed interval 9 s schedule of water reinforcement at ZT4. This operant schedule was used to control for the effects of nonattentional components of daily SAT practice, particularly lever pressing, reward rate, and associated motor activity (Arnold et al., 2002). Compared with SAT session, fixed interval $9 \mathrm{~s}$ (FI-9)-trained animals receive higher number of water rewards (below) and produce a $\sim 10$-fold greater number of lever presses [Arnold et al. (2002), their Fig. 2]. Thus, in the present context, evidence from animals performing this schedule served to test the possibility that the motor activity of SAT-performing rats contributes to their diurnality and to task period-synchronized elevations in cholinergic neurotransmission.

Animals were initially shaped to lever press on a modified FR-1 schedule for water reinforcement with only a single lever available (chambers equipped with red LED houselights). Following at least $3 \mathrm{~d}$ of 120 or more reinforced lever presses, animals started the FI-9 schedule for water reinforcement. To equate the duration of the session of animals performing the SAT, the adaptation period was lengthened to $10 \mathrm{~min}$. After an initial period of adaptation to the operant chambers (house lights on), a single lever was made available for responses. As this task was designed to minimize demands on attention that may result from tracking the presentation and removal of the response levers, this lever (left or right; counterbalanced across animals) remained extended for the entire length of the session. Animals underwent daily practice for at least 12 weeks to match the training period required for SAT performance. Following this extended training, animals underwent surgery for cannula implant in the mPFC (below) and allowed to recover for $48 \mathrm{~h}$ before FI- 9 training resumed for at least 5 more days and then animals were moved to concentric bowls for microdialysis (below).

Nonperforming animals. To determine whether there was a difference between basal ACh release across circadian phases, this control group was handled twice a week at randomly selected times to minimize entrainment effects. However, these animals were neither water-deprived nor performed an operant procedure [henceforth termed "nonperforming"
(NP)]. Consistent with the FI-9 control group, these animals were maintained under these conditions for at least 12 weeks before undergoing surgery and microdialysis procedures.

Food entrainment. To determine whether SAT performance period synchronized increase in prefrontal cholinergic neurotransmission was associated with SAT performance-entrained activity, a separate group of rats were first habituated for 1 week (as described above) and then food deprived for $28 \mathrm{~h}$, beginning with light onset (ZT0). Food was made available for a $3 \mathrm{~h}$ interval, beginning at ZT4, every day for 3 weeks. Water was available ad libitum. This procedure [modified from Mistlberger et al. (2009)] robustly entrains motor activity (Mendoza et al., 2005) and thus served as an additional test for the hypothesis that the specific demands of SAT performance were responsible for post-SAT practicesynchronized elevations on cholinergic neurotransmission. Following the 3 week interval, animals underwent surgery for cannula implant (below) and allowed to recover for $48 \mathrm{~h}$ with ad libitum access to food and water. Before being transferred to the dialysis chambers, the food restriction schedule resumed for least $5 \mathrm{~d}$.

Implantation of guide cannula for the measurement of mPFC ACh release. Animals underwent surgery to implant a guide cannula in the right $\mathrm{mPFC}$. Surgery was performed under aseptic conditions. Animals were anesthetized initially with $4-5 \%$ isoflurane in an anesthetic chamber (Anesco/Surgivet). Isoflurane was carried via oxygen at a flow rate of 0.6 $\mathrm{ml} / \mathrm{min}$. Animals' heads were shaved using electric clippers and cleaned with an alcohol wipe, they were then mounted to a stereotaxic instrument (David Kopf), and ophthalmic ointment was used to lubricate animals' eyes. Isoflurane was administered via a face mask, and levels were adjusted to $1.5-2 \%$ for the remainder of surgery. Microdialysis guide cannulas (model MAB 4.15.IC; Microbiotech) were implanted above the $\mathrm{mPFC}$ using the coordinates measured from bregma (in $\mathrm{mm}$ ): AP: +2.9 ; ML: \pm 0.6 ; DV: -0.6 below dura. To prevent clogging, the guide cannulas were equipped with stainless-steel stylets. A headstage to hold the guide cannula in place was fixed in place using surgical screws implanted into the skull and dental cement. Animals were given injections of an antibiotic (amikacin; $0.1 \mathrm{ml}$, i.p.) and an analgesic (buprenorphine; $0.01 \mathrm{mg} \cdot \mathrm{kg}^{-1} \cdot \mathrm{ml}^{-1}$, i.p.). The area around the headstage was coated with neosporin. Following surgery, animals were returned to their home cages and given ad libitum access to food and water while they recovered for $48 \mathrm{~h}$.

Microdialysis methods and determination of ACh concentrations. Dialysates were collected for $3 \mathrm{~h}$, beginning $90 \mathrm{~min}$ before the onset of the target period (e.g., period of prior SAT performance). Dialysates were collected on the third day after the last operant practice session (SAT or FI-9) in a new environment (concentric dialysis bowls; BASi). During the $3 \mathrm{~d}$ ending with the microdialysis session, the LD cycle matched the previous training conditions, and food and water were available ad libitum. Microdialysis sessions began with the removal of the stylet and the insertion of a removable concentric probe with a $3.0 \mathrm{~mm}$ membrane tip [model MAB 4.15.3; membrane outer diameter (o.d.), $0.2 \mathrm{~mm}$; shaft o.d., $0.18 \mathrm{~mm}$; Microbiotech] into the PFC. Animals were perfused at a rate of $2.0 \mu \mathrm{l} / \mathrm{min}$ with artificial CSF (aCSF), $\mathrm{pH} 6.8 \pm 0.1$, containing the following (in mM): $126.5 \mathrm{NaCl}, 27.5 \mathrm{NaHCO}_{3}, 2.4 \mathrm{KCl}, 0.5 \mathrm{Na}_{2} \mathrm{SO}_{4}, 0.5$ $\mathrm{KH}_{2} \mathrm{PO}_{4}, 1.2 \mathrm{CaCl}_{2}, 0.8 \mathrm{MgCl}_{2}$, and 5.0 dextrose. Dialysates were collected every $15 \mathrm{~min}$ for $180 \mathrm{~min}$. The ACh levels in the two collections obtained from intervals preceding the (prior) task time were averaged to determine basal ACh release. As a control condition, collections over 180 min were also obtained during the equivalent period $12 \mathrm{~h}$ later. Animals were then removed from the dialysis chambers, microdialysis probes were removed, stylets were reinserted, and animals were returned to their home cages. Dialysate samples were stored at $-80^{\circ} \mathrm{C}$ until they were analyzed by HPLC with electrochemical detection (ESA). The mobile phase used contained $35 \mathrm{~mm}$ sodium phosphate, $0.43 \mathrm{~mm}$ ethylenediamine tetra-acetic acid tetrasodium salt and $5 \mathrm{ml} / \mathrm{L}$ ProClin (BASi). ACh was separated from choline on a $250 \mathrm{~mm}$ analytical column and catalyzed on a postcolumn solid-phase reactor containing acetylcholinesterase and choline oxidase. ACh was then hydrolyzed to acetate and choline, and choline oxidized to hydrogen peroxide and betaine. The amount of hydrogen peroxide corresponding to ACh was then detected using a "peroxidase-wired" glassy carbon electrode with an applied potential of 
$-200 \mathrm{mV}$. To calculate the concentration of ACh in each sample, the integral of the area under the peak was taken and fit to a regression line containing known values of $\mathrm{ACh}$ in the expected range of the in vivo dialysates. The in vitro recovery of probes varied from 8 to $15 \%$. The detection limit of this system averaged $5 \mathrm{fmol} / 15 \mu \mathrm{l}$.

Lesion of basal forebrain cholinergic neurons. To determine the necessity of task period-synchronized increases in cholinergic activity for maintaining diurnal activity patterns, we lesioned the cholinergic projections to cortical regions using the immunotoxin 192 IgG saporin (192-SAP; Advanced Targeting System) in animals that reached stable SAT performance (McGaughy et al., 1996, 2000). The toxin or aCSF was infused bilaterally ( $200 \mathrm{ng} / \mu \mathrm{l} ; 0.8 \mu \mathrm{l} / \mathrm{hemisphere)}$ into the region of the nucleus basalis and substantia innominata of the basal forebrain (relative to bregma: AP, $-0.5 \mathrm{~mm}$; ML, $\pm 2.9 \mathrm{~mm}$; DV,$-7.5 \mathrm{~mm}$ from skull). The needle was left in position for $10 \mathrm{~min}$ to foster absorption of the toxin.

Histological verification of probe placement and cholinergic lesion. Following completion of the experiments, animals were deeply anesthetized and transcardially perfused with phosphate buffer solution followed by $4 \%$ paraformaldehyde in $0.1 \mathrm{~m} \mathrm{PBS,} \mathrm{pH}$ 7.4. Brains were removed and postfixed overnight at $4^{\circ} \mathrm{C}$, and then stored in $30 \%$ sucrose in $0.1 \mathrm{M}$ PBS and allowed to sink. Coronal sections $(40 \mu \mathrm{m})$ of the brains were sliced using a freezing microtome (CM 2000R; Leica) and stored in 0.1 м PBS until additional processing. The sections surrounding and including the probe site were mounted onto gelatin-coated glass slides and allowed to dry completely before being stained for Nissl substance and examined for probe placements.

Parallel sections were processed for the histochemical visualization of BF choline acetyltransferase (ChAT)-immunostained sections. ChAT immunostaining was accomplished by using a Vectastain Elite ABC kit (PK-6105; Vector Laboratories) and a primary antibody (polyclonal goat anti-choline acetyltransferase; Millipore). An orbital shaker was used throughout incubation and rinse periods. Sections were first rinsed in 0.1 M phosphate buffer, $\mathrm{pH} 7.4$, three times for $5 \mathrm{~min}$ each, and were then incubated in $0.3 \%$ peroxide for $30 \mathrm{~min}$. They were rinsed again in $0.1 \mathrm{M}$ phosphate buffer two times for $5 \mathrm{~min}$ each. After rinsing, sections were then incubated for $1 \mathrm{~h}$ in a $1.5 \%$ normal blocking serum with $0.2 \%$ Triton $\mathrm{X}$. After blocking, tissue was immediately transferred to incubate in the primary antibody (goat anti-ChAT made in rabbit; 1:250) overnight at $4^{\circ} \mathrm{C}$. The next day, they were rinsed three times for $5 \mathrm{~min}$ each in $0.1 \mathrm{M}$ PBS with $0.2 \%$ Triton X. They were then incubated in the biotinylated secondary antibody (biotinylated rabbit anti-goat; 1:200; supplied in the Vectastain Elite ABC kit) for $2 \mathrm{~h}$. After being rinsed three times for $5 \mathrm{~min}$ each in $0.2 \%$ Triton $\mathrm{X}$ in $0.1 \mathrm{M}$ phosphate buffer, tissue was incubated with the avidin-biotin complex (1:25) for $30 \mathrm{~min}$. They were rinsed three times for $5 \mathrm{~min}$ each in $0.1 \mathrm{M}$ phosphate buffer. Tissue was then rinsed in a peroxidase substrate solution of $0.4 \% \mathrm{DAB}$ (diaminobenzidine) and $0.19 \%$ nickel (II) chloride in $0.1 \mathrm{M}$ phosphate buffer. Ten microliters of $30 \%$ hydrogen peroxide were added immediately before use. Once sections reached a desired color $(\sim 5 \mathrm{~min})$, they were rinsed with $0.1 \mathrm{M}$ phosphate buffer three times for $5 \mathrm{~min}$ each. Omission of the primary antibody resulted in no specific staining, supporting the specificity of this protocol. Sections were mounted on gelatin-coated slides and were allowed to dry overnight. The following day, slides were dehydrated in an ascending alcohol series (70,90, and 100\%) and defatted in xylene before coverslipping.

Statistical methods. Because of the complexity of the designs used for the multiple experiments described below, the main factors and statistical methods are identified in the description of results. Generally, mixeddesign ANOVAs were used to determine the effects of previous SAT training on prefrontal cholinergic neurotransmission of intact and lesioned animals, and to determine the effects of BF lesions on SAT performance. Repeated-measures ANOVA included the collections over the intervals coinciding with the previous pretask and task blocks (block1block 3 ) as no differences were found in the collections obtained from posttask intervals. Post hoc multiple comparisons were conducted using $t$ test and Fisher's least significant difference (LSD) test. $\alpha$ was set at 0.05 . Post hoc multiple comparisons were conducted using $t$ test and Fisher's LSD. Statistical analyses were performed using SPSS for Windows (version 17.0; SPSS Inc.). In cases of violation of the sphericity assumption,
Huyhn-Feldt-corrected $F$ values, along with uncorrected degrees of freedom, are given. Exact $p$ values are reported (Greenwald et al., 1996).

\section{Results}

\section{SAT performance time-stamped ACh release}

We reported previously that daily practice of a SAT during the light phase, even if limited to short 8 min practice periods, generates a diurnal activity pattern in rats. Neither daily practice of operant control procedures not taxing attention nor of a water maze yielded such diurnality (Gritton et al., 2009, 2012). Following termination of daily SAT practice, such diurnality persisted for at least for $4 \mathrm{~d}$ (see also below). The first experiment was guided by the hypothesis that, as a result of prior SAT practice in the light phase, relatively higher levels of ACh release levels are observed during the light when compared with the dark phase, thereby reversing the regular circadian regulation of cholinergic activity [higher levels in the dark phase (Kametani and Kawamura, 1991; Jiménez-Capdeville and Dykes, 1996)]. Instead, we found that increases in cholinergic neurotransmission during the light phase were precisely timed to the prior period of SAT practice, but not to the period of practicing an operant schedule of reinforcement.

Figure $1 a$ illustrates the timeline and major events for this first set of experiments. For animals performing the SAT beginning $4 \mathrm{~h}$ into the light phase (ZT4), Figure $1 b$ shows the relative number of hits across the three signal durations (averaged over the last three SAT sessions). For comparison, this figure also shows the equivalent hit rates during the last three sessions of animals practicing the task beginning at $4 \mathrm{~h}$ into the dark phase (ZT16) and in animals that were maintained under constant-light conditions (LL) (below). Hit rates generally varied with signal duration $\left(F_{(2,30)}=124.34 ; p<0.001\right)$ but did not differ across groups [ZT4, ZT16, LL: $F_{(2,15)}=2.83 ; p=0.09$; mean (M), SEM: $64.16 \pm$ $2.87 \%$; group by duration: $\left.F_{(4,30)}=4.48 ; p=0.006\right]$; the interaction reflected a significantly lower detection rate of shortest signals by LL animals when compared with ZT16 but not ZT4 animals (group: $F_{(2,17)}=5.57 ; p=0.02$; LL vs ZT16: $t_{(10)}=5.17$; $p<0.001)$. Response accuracy for nonsignal trials did not differ between the groups (group: $F_{(2,15)}=3.51 ; p=0.056$; M, SEM: $82.03 \pm 1.86 \%$ correct rejections) and animals generally omitted very few trials (group: $F_{(2,15)}=1.35 ; p=0.29 ; 2.33 \pm 1.05 \%$ ). Animals performing the FI-9 schedule of reinforcement (also beginning at ZT4) generated $965.88 \pm 120.84$ lever presses per session, significantly more than the number of lever presses occurring during SAT sessions $\left(202.00 \pm 1.56 ; t_{(14)}=6.32 ; p<\right.$ $0.001)$. Similarly, the number of water reinforcements obtained per session was significantly higher in FI-9- compared with SATperforming animals (207.25 $\pm 1.47 ; 107.14 \pm 6.75$, respectively; $\left.t_{(14)}=7.25 ; p<0.001\right)$. This experiment also included animals that never performed a task (NP) but were handled at random periods (see Materials and Methods).

\section{Basal ACh release across the LD cycle in NP rats}

We first analyzed the absolute ACh levels obtained from nonperforming animals to confirm that basal ACh levels in the cortex of rats are generally higher during the nocturnal activity phase. Although this notion is widely accepted in the literature, the available evidence remains limited (for review, see Hut and Van der Zee, 2011) and, when based on in vivo measures using microdialysis, burdened by the addition of micromolar concentrations of an acetylcholinesterase inhibitor to the dialysis perfusion medium, thereby dampening presynaptic release (references above). Rats housed in a 12:12 LD cycle (nonperforming) exhibited a 

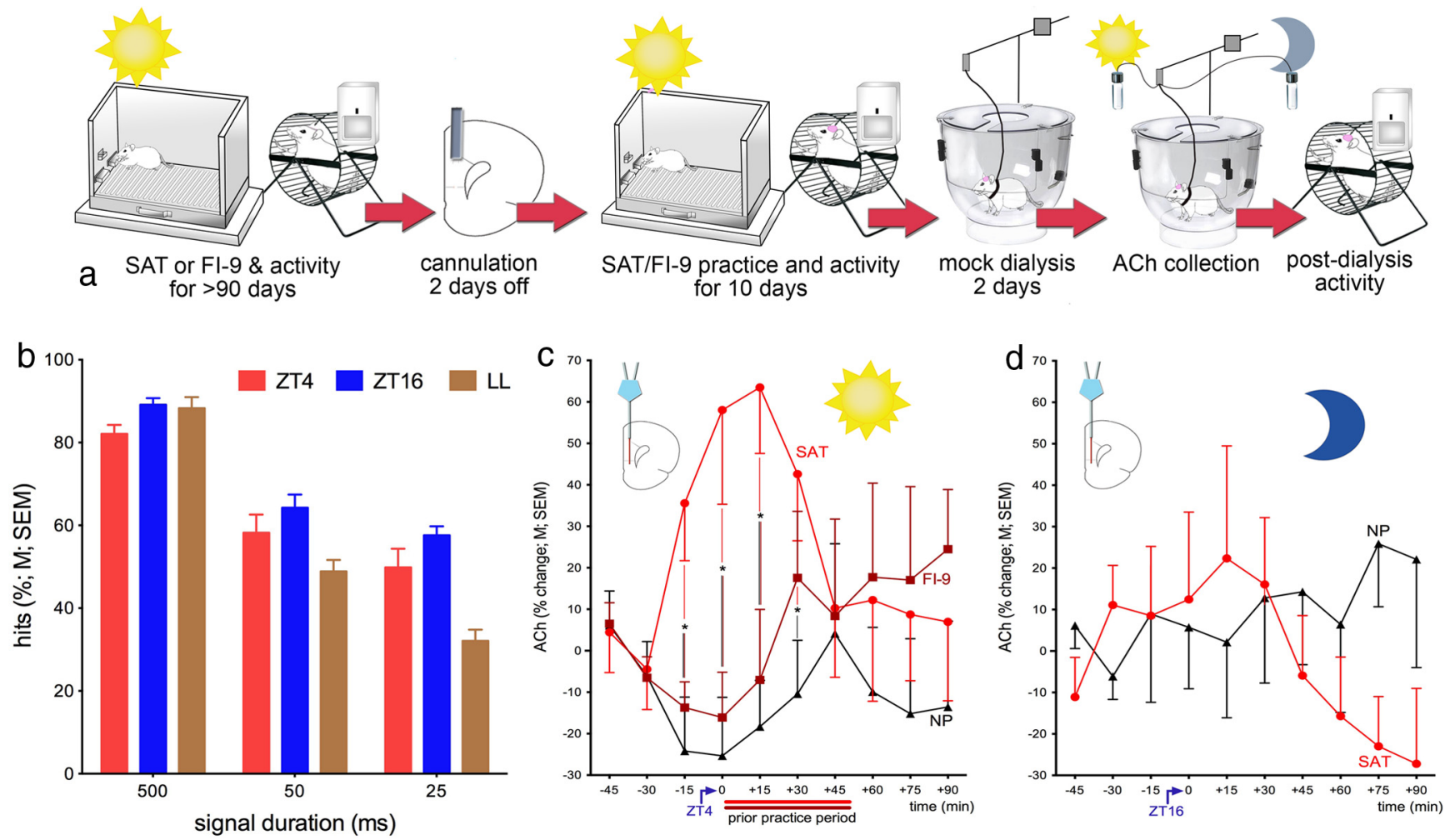

Figure 1. Daily practice of the SAT, beginning at $4 \mathrm{~h}$ into the light phase (ZT4), generates task time-stamped increases in prefrontal cholinergic activity. $\boldsymbol{a}$, Timeline and sequence of major experimental procedures and events. For at least $90 \mathrm{~d} /$ sessions, rats practiced the SAT $(N=21)$ or an operant control procedure that generated higher number of rewards and higher number of lever presses per session ( $\mathrm{Fl}-9$ schedule of reinforcement; $n=8$ ). Handled but NP animals $(n=7)$ constituted a second control group. Following implantation of a guide cannula for subsequent insertion of a microdialysis probe, performance practice and activity recording resumed for $10 \mathrm{~d}$. Animals were then transferred to live in microdialysis bowls, under mock-dialysis conditions (tethered but probe not inserted) for $2 \mathrm{~d}$. On day 3, medial prefrontal dialysates were collected (15 min each) for $3 \mathrm{~h}$ beginning $90 \mathrm{~min}$ before ZT4, and for an equivalent period $12 \mathrm{~h}$ later during the dark phase. On day 4 , animals were returned to their home cages and activity was recorded. $\boldsymbol{b}$, Relative number of hits over signal durations for SAT at ZT4 performing animals (red bars; $n=8$ ), averaged over the final three SAT sessions before microdialysis (see Results for additional measures of performance). Performance at ZT4 did not differ significantly from performance beginning $4 \mathrm{~h}$ into the dark phase (ZT16, blue bars; $n=8$ ) or while maintained in constant light (LL) (brown bars; $n=5 ;$ see Fig. 3 for ACh release data from ZT16 and LL animals). Error bars indicate SEM. c, Increases in ACh release in previously SAT-performing (ZT4) animals began $30-15$ min in advance to the prior task time and lasted four collections (60 min). Such increases were found neither in previously FI-9 performing animals nor in nonperforming controls (for ANOVAs, see Results; post hoc multiple comparisons: ${ }^{*} p<0.05$ ). $\boldsymbol{d}$, Prior SAT performance did not generally alter the regulation of cholinergic activity as indicated by the absence of systematic changes in ACh release over 10 collections taken during the dark phase $12 \mathrm{~h}$ later (light and dark phases are symbolized by sun and moon symbols).

regular nocturnal activity pattern (LD ratio: $0.22 \pm 0.05$ ). ACh release levels showed a trend for being higher during the dark phase (main effect of phase, $F_{(1,6)}=5.82 ; p=0.052$; dialysates obtained from ZT14.5 through ZT17.5: $8.24 \pm 2.23 \mathrm{fmol} / 30 \mu \mathrm{l}$; ZT2.5-ZT5.5: $4.50 \pm 1.60 \mathrm{fmol} / 30 \mu \mathrm{l})$. There was neither an effect of collection nor an interaction between phase and collection (both $F_{(9,54)}<1.65$; both $p>0.12$ ), indicating that ACh release levels remained stable within phase (light or dark).

\section{Cholinergic activation time-stamped to prior SAT, but not}

FI-9, practice

As illustrated in Figure 1a, animals underwent microdialysis for the determination of mPFC ACh release $3 \mathrm{~d}$ after the final SAT or FI-9 session. ACh was collected for a period beginning $45 \mathrm{~min}$ before ZT4 and for an additional 90 min thereafter.

Basal ACh release did not differ in rats that previously performed the SAT or the FI-9 schedule of reinforcement $\left(F_{(1,10)}=\right.$ $0.000 ; p=0.99 ; 4.75 \pm 0.45 \mathrm{fmol} / 30 \mu \mathrm{l})$. Therefore, ACh concentrations were expressed as percentage change from baseline (Fig. 1c). Prefrontal ACh release began to rise $15 \mathrm{~min}$ before the previous onset of the SAT at ZT4, peaked at 15 min into the prior task period, and returned to baseline levels at the time the prior SAT practice had ended. No such rise in cholinergic activity was found in animals that had performed the FI-9 operant schedule or in nonperforming animals. A mixed-design ANOVA con- firmed that levels of ACh release differed by group $\left(F_{(2,20)}=3.67\right.$; $p=0.044$; for multiple comparisons, see Fig. 1c). This analysis also indicated an interaction between the effects of group and collection interval $\left(F_{(18,180)}=2.20 ; p=0.012\right.$; no main effect of time, $p=0.73$ ), indicating that changes in ACh release occurred exclusively in animals that had previously performed the SAT.

To further explore the validity of this interpretation, one-way ANOVAs were computed over the effects of collection intervals (or time) on ACh release obtained throughout the prior performance period. These analyses indicated a significant effect of block on ACh levels in animals that had performed the SAT (SAT: $\left.F_{(6,42)}=2.88 ; p=0.032\right)$ but not the operant control procedure (FI-9 and NP: both $p>0.33$ ). Multiple comparisons confirmed that ACh levels were significantly higher in the first two collections into the prior task period $(+15,+30 \mathrm{~min})$ than at -45 and $-30 \mathrm{~min}$ (Fig. 1c), and that the collection ending with ZT4 contained more ACh than the -30 min collection. Collectively, this evidence indicates that, $3 \mathrm{~d}$ after the final SAT session, and while housed in a different environment, mPFC cholinergic neurotransmission was elevated for $45 \mathrm{~min}$, matching the prior task duration. Furthermore, the initial increase in cholinergic activity occurred $15 \mathrm{~min}$ (or one dialysate collection) before ZT4, therefore also covering the $15 \mathrm{~min}$ pretask period during which animals were transferred to the operant chambers. 

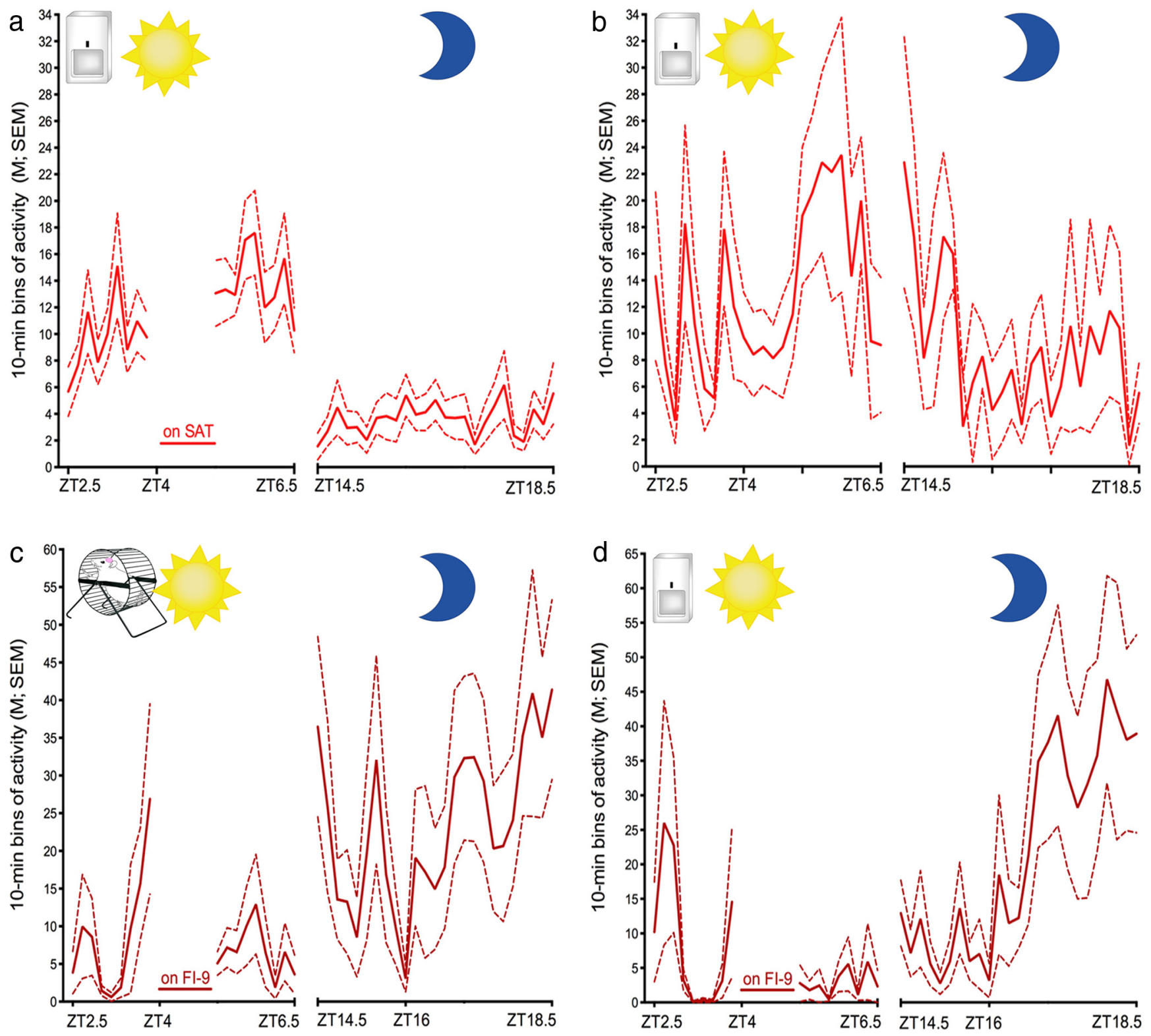

Figure 2. Daily practice of the SAT, beginning at $4 \mathrm{~h}$ into the light phase (ZT4), generates a diurnal activity pattern. $\boldsymbol{a}$, Circadian activity pattern of rats practicing the SAT at ZT4, averaged over the final three task sessions (see Results for statistical analyses; the top left symbol indicates IR device-based measures). These rats exhibited a stable diurnal activity pattern. $\boldsymbol{b}$, Circadian activity after completion of the microdialysis experiments, $4 \mathrm{~d}$ after the final SAT session. Animals maintained a diurnal activity pattern, albeit to a lesser degree than on days involving final SAT sessions. The rats' circadian activity pattern was recorded either via IR motion detectors or running wheel. $\boldsymbol{c}, \boldsymbol{d}$, Activity scores obtained from either method support the conclusions. Circadian activity pattern of rats during their last $3 \mathrm{~d}$ of performing the FI-9 schedule of reinforcement at ZT4, assessed with either wheels ( $\boldsymbol{c}$ ) and IR devices (d). Although activity increased in anticipation of the task period, rats maintained a stable nocturnal activity pattern [see also SAT at ZT4 induced diurnality as measured by IR device (Fig. 2a) or by wheels (Fig. 5b)].

ACh release 12 h later

To determine whether the diurnal activity pattern that results from the SAT performance at ZT4 (below) was associated with a potentially global reorganization in the pattern and dynamics of mPFC cholinergic neurotransmission, dialysates were also collected during an equivalent interval $12 \mathrm{~h}$ later in the dark phase and compared with ACh release levels from NP rats. Absolute ACh levels during the first two collections of previously SAT performing animals were $3.87 \pm 0.71 \mathrm{fmol} / 30 \mu \mathrm{l}$ and did not differ from those detected in the first two collections obtained during the daytime period $\left(4.58 \pm 1.02 \mathrm{fmol} / 30 \mu \mathrm{l} ; t_{(5)}=0.65\right.$; $p=0.54)$. ANOVA did not indicate effects of group or collection interval (effects of group, collection, and interaction: all $F<0.79$; all $p>0.54$; Fig. $1 d)$.

\section{Circadian activity pattern}

Consistent with our prior findings (Gritton et al., 2009, 2012), and while still performing the SAT at ZT4 (last three sessions; Fig. $1 a$ ), rats exhibited a diurnal activity pattern (Fig. $2 a$; LD ratio, $1.97 \pm 0.34$; significantly different from the LD ratio of NP rats, $0.22 \pm 0.05 ; t_{(10)}=4.09 ; p=0.009$; activity counts of the two phases: light phase, $11.79 \pm 0.59$; dark phase, $3.38 \pm 0.35 ; t_{(7)}=$ $3.88 ; p=0.006)$. Such a diurnal activity pattern persisted and was again observed, albeit to a lesser and, with respect to activity counts, insignificant degree, $1 \mathrm{~d}$ after the dialysis collections, that is, $4 \mathrm{~d}$ after the final SAT session (Fig. $2 b$; LD ratio, $1.04 \pm 0.9$; activity counts: light phase, $12.99 \pm 1.27$; dark phase, $9.11 \pm 1.08$; $\left.t_{(7)}=1.96 ; p=0.09\right)$. In contrast, practicing the FI-9 schedule of reinforcement at ZT4 was not associated with the presence of a 

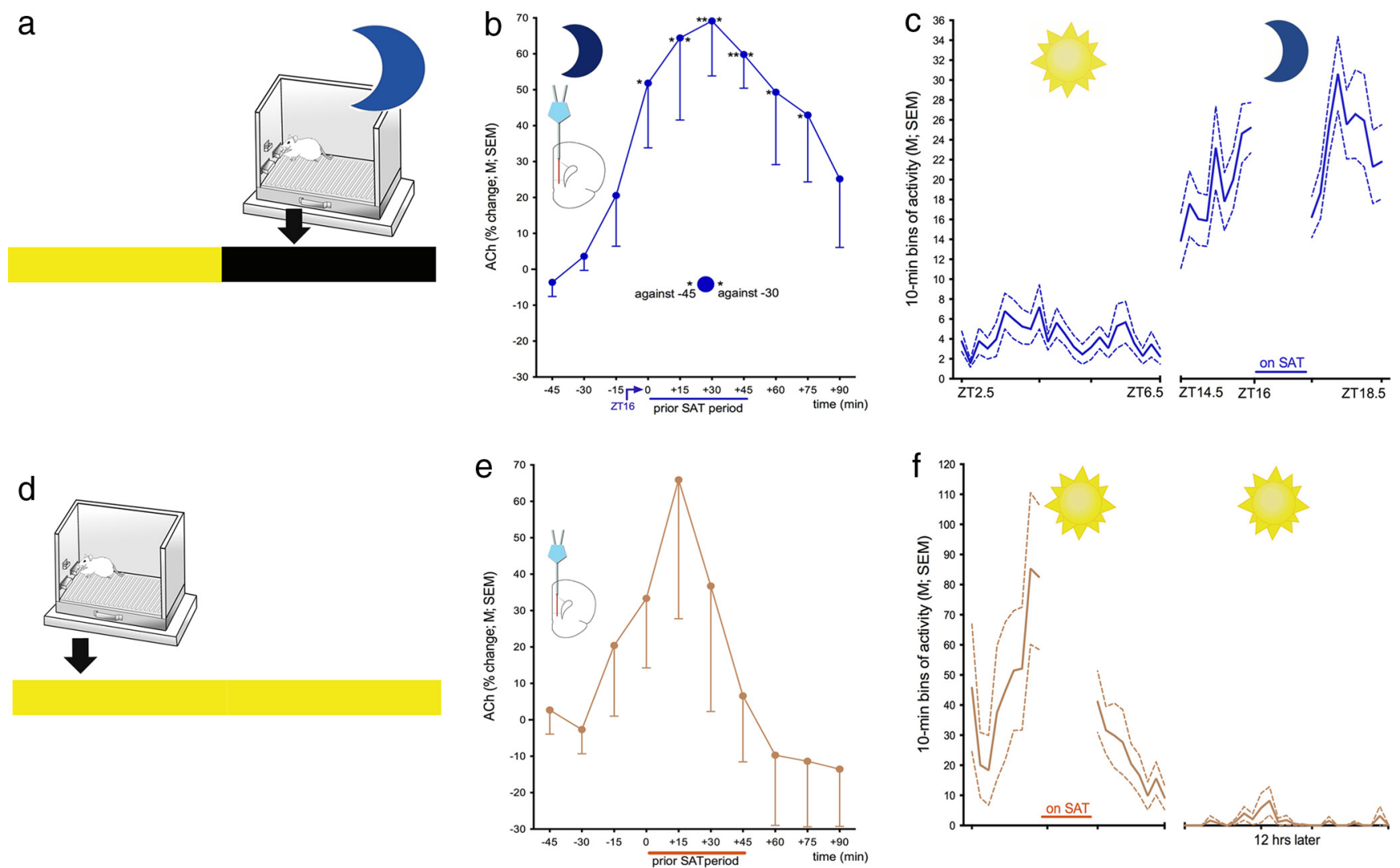

Figure 3. Task time-stamped increases resulting from SAT practice in the dark phase, beginning at ZT16, and by animals maintained under constant-light conditions (LL). $\boldsymbol{a}$, Except for SAT practice beginning $4 \mathrm{~h}$ into the dark phase $(Z T 16 ; n=8)$, the experimental procedures and sequence of events followed the illustration in Figure 1a. $\boldsymbol{b}$, Increases in prefrontal cholinergic neurotransmission, $3 \mathrm{~d}$ after the final SAT session, anticipated the prior task period by one collection ( $15 \mathrm{~min}$ ) and persisted for two collection intervals ( $30 \mathrm{~min}$ ) beyond the prior task time ( $p o s t$ hoc multiple comparisons: $\left.{ }^{*} p<0.05,{ }^{* *} p<0.001\right)$.c, As would be expected, SAT practice at ZT16 did not alter the nocturnal activity pattern, which peaked right before and after task time.d, Daily SAT practice in animals kept in LL ( $n=5$ ) occurred at a fixed time. e, Subsequent increases in prefrontal ACh release in LL animals peaked 15 min into the prior task time. Because of the greater variability of ACh release in LL animals, multiple comparisons did not locate the main effect indicated by ANOVA (see Results). Thus, while task time-stamped cholinergic activation did not depend on the presence of the LD cycle, the absence of photic zeitgebers may have contributed to the greater variability of ACh release. $f$, Prior daily SAT practice entrained an activity pattern that peaked around task time, with little activity during the subsequent $12 \mathrm{~h}$ period.

diurnal activity pattern (Fig. $2 c$; LD ratio, $0.32 \pm 0.06$; activity counts: light phase, $7.72 \pm 1.26$; dark phase, $23.57 \pm 2.01 ; t_{(7)}=$ $3.14 ; p=0.016)$. Furthermore, activity patterns monitored by running wheels (Fig. $2 c$ ) or IR motion detectors (Fig. $2 d$ ) both indicated identical nocturnal activity patterns of previously FI-9 performing animals (method: $F_{(1,6)}=0.017 ; p=0.09$; circadian phase: $F_{(1,6)}=7.85 ; p=0.031$; interaction: $F_{(1,6)}=$ $0.41 ; p=0.54)$.

\section{ACh release time-stamped to prior SAT practice at ZT16 and}

in $L L$

SAT performance-period synchronized ACh release in animals that had practiced at ZT4 and therefore developed a diurnal activity pattern may depend on interactions between diurnality and the time of prior task practice, as opposed to a generally synchronized activation of cholinergic neurotransmission at the time of the prior fixed daily practice of an attention-demanding task. The former hypothesis would predict that synchronized ACh release is not observed in animals that practiced the SAT beginning $4 \mathrm{~h}$ into the dark phase (ZT16).

As illustrated in Figure $3 b$, prefrontal cholinergic neurotransmission increased at precisely the time of the prior SAT practice at ZT16 $\left(F_{(9,63)}=2.39 ; p=0.009\right.$; see Fig. $3 b$ for multiple comparisons). Notably, the onset of this increase in cholinergic activity was, by $15 \mathrm{~min}$, even more precisely synchronized to the onset of prior SAT practice than was the case after practice at ZT4.
However, ZT16-stamped cholinergic activity lasted for $90 \mathrm{~min}$ ( six collections), and thus for 30 min longer than in ZT4 animals. Prior ZT16 practice was associated with a pronounced nocturnal activity pattern (LD ratio, $0.41 \pm 0.05$; activity counts: light phase, $4.00 \pm 0.34$ counts; dark phase, $21.46 \pm 0.79$ counts; $t_{(7)}=$ 8.03; $p<0.001$; Fig. $3 c$ ).

To further substantiate that SAT performance timesynchronized ACh release did not depend on the presence of a $12 \mathrm{~h} \mathrm{LD}$ cycle, we also measured ACh release in animals maintained in LL and that had developed a free running rhythm [period length $(\tau)$ : before LL and while still in LD, $24.04 \pm 0.02 \mathrm{~h}$; LL, $24.81 \pm 0.17$; after reaching SAT criterion in LL, $23.99 \pm 0.022$; $\left.F_{(2,8)}=7.13 ; p=0.018\right]$; multiple comparisons indicated that the animals' period lengthened as they were moved from LD to LL $\left(t_{(4)}=4.36 ; p=0.012\right.$ ), consistent with prior reports (Pittendrigh and Daan, 1976). Similar to ACh release in animals that had previously practiced the SAT at ZT4 or ZT16, prior SAT performance in LL produced significant increases in cholinergic neurotransmission, peaking at a time corresponding to the prior first task block $\left(F_{(9,36)}=2.67 ; p=0.046\right)$. However, ACh release in LL rats was more variable than in the other two groups, preventing multiple comparisons from locating the effect (Fig. 3e). Inspection of individual release data did not suggest that this variability reflected a less precise timing of peak ACh release levels; indeed, all ACh peaks in LL animals occurred at ZT4, +15 or +30 min. As 

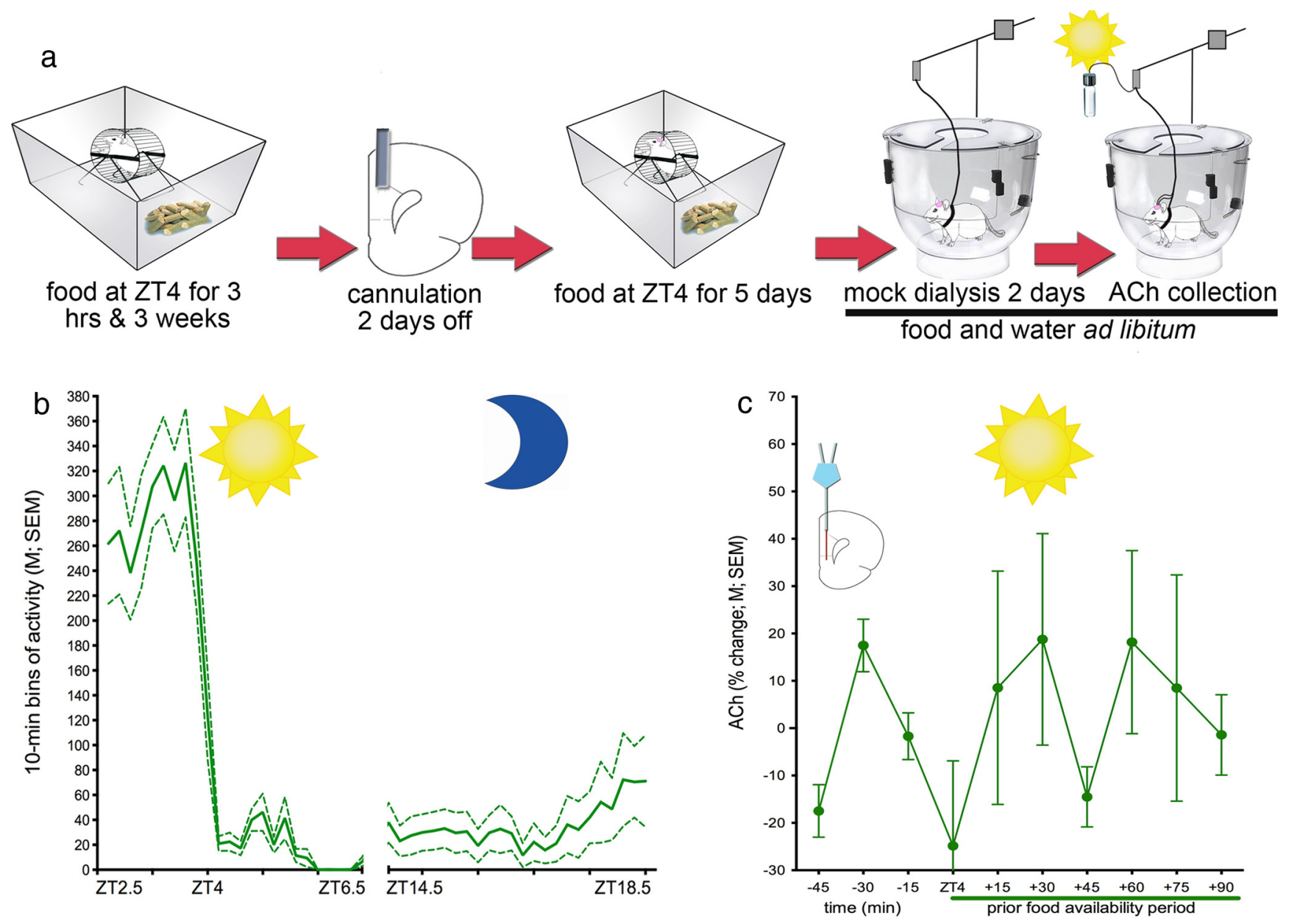

Figure 4. FAA was not associated with time-stamped increases in cholinergic activity. $\boldsymbol{a}$, Timeline and major experimental events. Animals were habituated to the restricted feeding schedule, with food available for $3 \mathrm{~h}$ beginning at ZT4, for 3 weeks $(N=7)$. Following $2 \mathrm{~d}$ of postsurgery recovery, the restricted feeding regimen resumed for $5 \mathrm{~d}$. As before, ACh was collected following acclimation to the dialysis chambers, with food and water available ad libitum. $\boldsymbol{b}$, As expected, FAA was indicated by robust anticipatory wheel running followed by little activity during the feeding period. c, FAA was not associated with anticipatory of feeding period-associated increases in cholinergic neurotransmission.

would be expected based on the diurnal activity pattern in ZT4 animals, animals that had practiced the SAT in LL exhibited an activity pattern that increased toward the task time while showing very little activity during the subsequent $12 \mathrm{~h}$ period $(12 \mathrm{~h}$ task/12 h no-task ratio, $3.01 \pm 0.51$; activity counts: $t_{(5)}=2.71 ; p=$ 0.042; Fig. $3 f$ ).

Food-anticipatory activity at ZT4 is not associated with increases in cholinergic neurotransmission

All animals, with the exception of nonperforming rats in the first experiment, were water-deprived and received water as reward during the task and for a limited period thereafter (see Materials and Methods). We previously demonstrated that diurnal circadian entrainment associated with the SAT is not caused by daily timed water access (Gritton et al., 2009). However, foodanticipatory activity (FAA) represents one of the most extensively studied zeitgebers (Boulos et al., 1980; Mistlberger, 1994; Stephan, 2002; Mendoza et al., 2005). Therefore, a separate group animals was made extensively familiar, for 3 weeks, with rat chow made available for $3 \mathrm{~h}$ beginning at ZT4 (water was available ad libitum). Note that restricting periods of food availability to an even shorter period, corresponding with the 45 min of task time, was not permissible because of significant and even vital weight loss (Treichler and Hall, 1962). Animals were dialyzed after the feeding regimen was reinstituted for $5 \mathrm{~d}$ after surgery and after $2 \mathrm{~d}$ of acclimation in the dialysis bowls, with food and water now available ad libitum.

As was expected, these rats exhibited strong FAA. Because most of the animals' activity occurred during the anticipatory phase, close to ZT4, with little activity during the feeding phase, and because activity during the dark phase was not attenuated (Fig. 4b), the regular LD analysis (across the full $12 \mathrm{~h}$ phases) does not fully reflect the impact of FAA (LD ratio, $1.07 \pm 0.18$ ). A more restricted analysis comparing the activity counts obtained during the $1.5 \mathrm{~h}$ period before ZT4 and the equivalent period $12 \mathrm{~h}$ later (Fig. $4 b$ ), indicates the effect of FAA (FAA, 282.82 \pm 20.88 ; 12 h later, $\left.28.26 \pm 6.03 ; t_{(6)}=3.72 ; p=0.01\right)$. Importantly, ACh release did not change across the collections that were associated with FAA, with mean changes in ACh levels remaining below $20 \%$ above baseline $\left(F_{(9,27)}=0.80 ; p=0.44\right.$; Fig. $\left.4 c\right)$.

\section{Resuming nocturnality and loss of SAT time-stamped cholinergic activation}

The evidence from animals performing the SAT at ZT4 is consistent with the hypothesis that task practice period-synchronized increases in cholinergic neurotransmission contribute to the development and maintenance of a diurnal activity pattern. Furthermore, such diurnality and synchronized cholinergic activity patterns persisted for at least $3 \mathrm{~d}$ following the final SAT perfor- 
mance session (above). In this experiment, animals were equipped with guide cannula for microdialysis, underwent postsurgery SAT practice for $5 \mathrm{~d}$, and were then permanently housed in their home cages with food and water available ad libitum and access to running wheels. A nocturnal activity pattern was fully reestablished $8 \mathrm{~d}$ after the last SAT session (LD ratio, $0.19 \pm 0.07$; activity counts: light phase, $0.96 \pm 0.24$; dark phase, $14.3 \pm 0.71$; $\left.t_{(3)}=4.13 ; p=0.026\right)$. At that time, collection of dialysates in these animals (using the procedures illustrated in Fig. 1a) did not reveal significant changes in ACh levels across 10 collections over a time period that included the prior task practice time $\left(F_{(9,27)}=\right.$ $0.55 ; p=0.74)$.

\section{Above-chance SAT performance and associated cholinergic activity are necessary for maintaining time-stamped cholinergic activation and diurnal activity}

Removal of cortical cholinergic inputs persistently disrupts SAT performance (McGaughy et al., 1996; McGaughy and Sarter, 1998). This experiment tested the hypothesis that such removal and the resulting impairment in SAT performance prevent the expression of prior task time-synchronized increases in cholinergic activity and associated diurnality. Although this experiment does not allow the isolation of the individual effects of loss of cholinergic neurons and impaired SAT performance (but see Discussion), it was designed to potentially reject the overall hypothesis that SAT performance and underlying cholinergic activity contribute to the diurnal activity pattern that emerges during SAT training and practice.

Figure $5 a$ illustrates the timeline of major events of this relatively complex experiment. As before, animals performing the SAT at ZT4 exhibited a diurnal activity pattern [Fig. 5b; LD ratio, $2.17 \pm 0.45$; comparison with the LD of NP rats (above): $t_{(10)}=$ $4.31 ; p=0.007]$. Infusions of the immunotoxin into the basal forebrain removed $>80 \%$ of extrinsic cholinergic innervation of the cortex (Fig. $5 g-k$ ). As a result, SAT performance was robustly impaired (main effect of lesion on SAT scores: $F_{(1,6)}=18.74 ; p=$ 0.005; see Fig. $5 c$ for multiple comparisons). As illustrated in Figure $5 c$, SAT performance remained signal duration-dependent $\left(F_{(2,12)}=\right.$ 29.56 ; $p<0.001$; lesion by duration: $p=0.34$ ). Chance performance in this task consist of 50\% hits and 50\% correct rejections, with the threshold for statistically above chance performance at a SAT score of 0.17 or higher (see dashed line in Fig. $5 c$ ). Lesioned animals' performance scores calculated over hits to medium and shortest signals were below chance level.

After $60 \mathrm{~d}$ of postlesion SAT performance at such low levels, and before the microdialysis part of this experiment, lesioned animals continued to exhibit a diurnal activity pattern (LD ratio, $1.29 \pm 0.42$ ), although the more restricted analysis of activity counts did not reach significance (light phase, $11.48 \pm 1.42$; dark phase, $4.99 \pm 0.94 ; t_{(6)}=1.33 ; p=0.23$; Fig. $\left.5 d\right)$. At this point, neither the LD ratio $\left(t_{(13)}=-0.57 ; p=0.58\right)$ nor the light phase activity counts $\left(t_{(13)}=0.09 ; p=0.93\right)$ differed between lesioned and control animals. As would be expected, in deafferented animals, prefrontal ACh levels did not vary across the collection periods that included the prior task period beginning at ZT4 $\left(F_{(1,12)}=0.54 ; p=0.47 ;\right.$ Fig. $\left.5 e\right)$. Correlational analyses indicated a trend for lesioned animals with higher ACh levels at +15 minwhen controls exhibit highest release levels (Fig. 1c) - to exhibit relatively more diurnal activity (LD ratios; Pearson's $r=0.84$; $p=0.07)$. Finally, the activity patterns of these animals, recorded $1 \mathrm{~d}$ after the microdialysis session, had completely reverted to a nocturnal phenotype (LD ratio, $0.22 \pm 0.11$; activity counts: light phase, $0.80 \pm 0.25$; dark phase, $19.33 \pm 3.19$; Fig. $5 f$ ). This find- ing contrasts with the persistent diurnal activity pattern observed in intact animals recorded following an identical number of days (4) after the final SAT session (Fig. $2 b$; LD ratio, $t_{(13)}=5.81 ; p<$ 0.001 ; light phase activity counts: $t_{(13)}=4.33 ; p=0.003$; intact, $12.99 \pm 1.08$; lesioned, $0.08 \pm 0.25)$.

\section{Benefit of time stamping: shifting SAT practice time within the light cycle}

Circadian influences on skilled and cognitive performance have been extensively demonstrated (Folkard et al., 1983; Fekete et al., 1985; Gerstner and Yin, 2010). The findings described above indicate that attentional performance at a fixed time generates rather precisely synchronized increases in cholinergic neurotransmission. As such increases in cholinergic activity enhance the readiness of cortical circuitry for input processing (Edeline et al., 1994), this finding predicts that shifts in the timing of performance, even within phase, should detrimentally affect performance because of the absence of anticipatory and additive cholinergic activity (but see Discussion).

Because the potential detrimental performance effects of phase advancement (shifting to an earlier time) would be confounded by a shorter period of water deprivation (Echevarria et al., 2005), we tested the effects of a phase delay (shifts to a later time). As such a delay involved longer than usual periods of water deprivation, the enhanced motivated recruitment of the cholinergic attention system (St. Peters et al., 2011a) favored the rejection of the hypothesis that such a shift lowers performance. For this experiment, animals first practiced the SAT at ZT2 for at least $90 \mathrm{~d}$ and then shifted to SAT practice to ZT8. ANOVA of the SAT scores included the last three sessions prior and after the shift. The shift resulted in a significant decrease in SAT performance $\left(F_{(1,7)}=11.22 ; p=0.012\right.$; SAT scores: preshift, $0.49 \pm 0.03$; postshift, $0.40 \pm 0.03$; Fig. 6$)$. The effect of the phase delay did not interact with day, indicating that the performance was stable before the shift and did not recover during the first $3 \mathrm{~d}$ after the shift (main effects of day and interaction: both $p>0.48$ ). This is consistent with prior observations indicating that shifting SAT performance to a new time and even into a different phase requires weeks of practice for performance to return to preshift levels (Gritton et al., 2012). The shift-induced decrease in the SAT score was due to impairments in the performance of signal and nonsignal trials, neither of which reached significance if analyzed individually [hits (averaged over signal duration): preshift, $59.04 \pm 3.29 \%$; postshift, $52.76 \pm 3.58 \%$; correct rejections: pre, $86.17 \pm 0.98 \%$; post, $80.96 \pm 3.73 \%$; both $p>0.05$ ]. Errors of omission were not affected by this shift $\left(F_{(1,7)}=1.55 ; p=0.25\right)$ and remained generally low $(<10 \%)$.

\section{Discussion}

The following main findings were obtained from five series of experiments. (1) Daily practice of a SAT at a fixed time, but not of an operant schedule of reinforcement, resulted in relatively precise task time-synchronized increases in prefrontal cholinergic neurotransmission. These time-stamped increases in cholinergic neurotransmission were measured after animals were taken off task and maintained in a new environment. Furthermore, these time-stamped elevations in prefrontal cholinergic activity were observed regardless of whether the SAT was practiced during the light or dark period or while placed in constant-light conditions (LL). (2) Animals practicing the SAT beginning at $4 \mathrm{~h}$ into the light phase (ZT4) maintained a diurnal activity pattern. No such diurnality was observed in animals practicing the FI-9 operant schedule of reinforcement despite the robustly higher reward and 

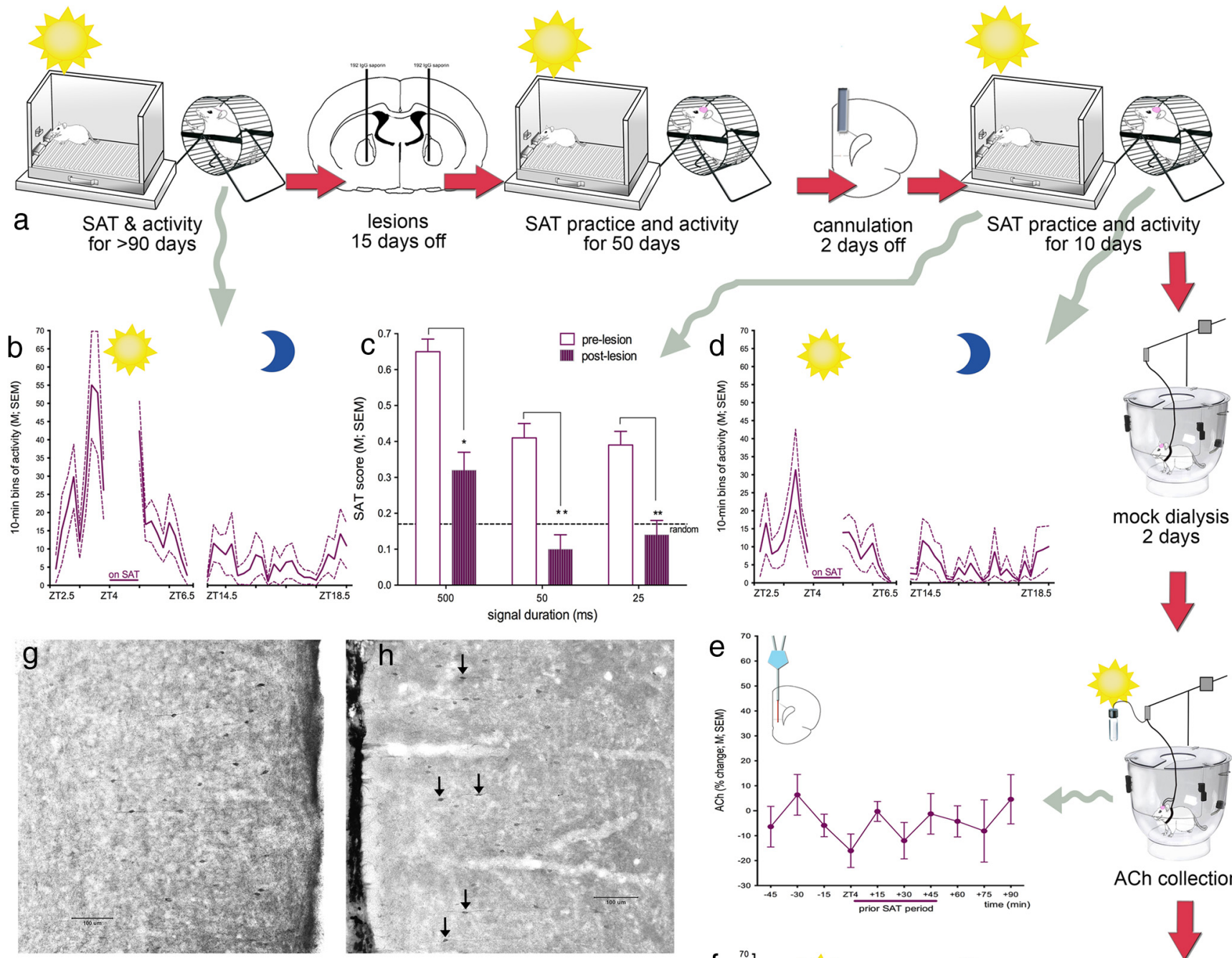

mock dialysis

2 days
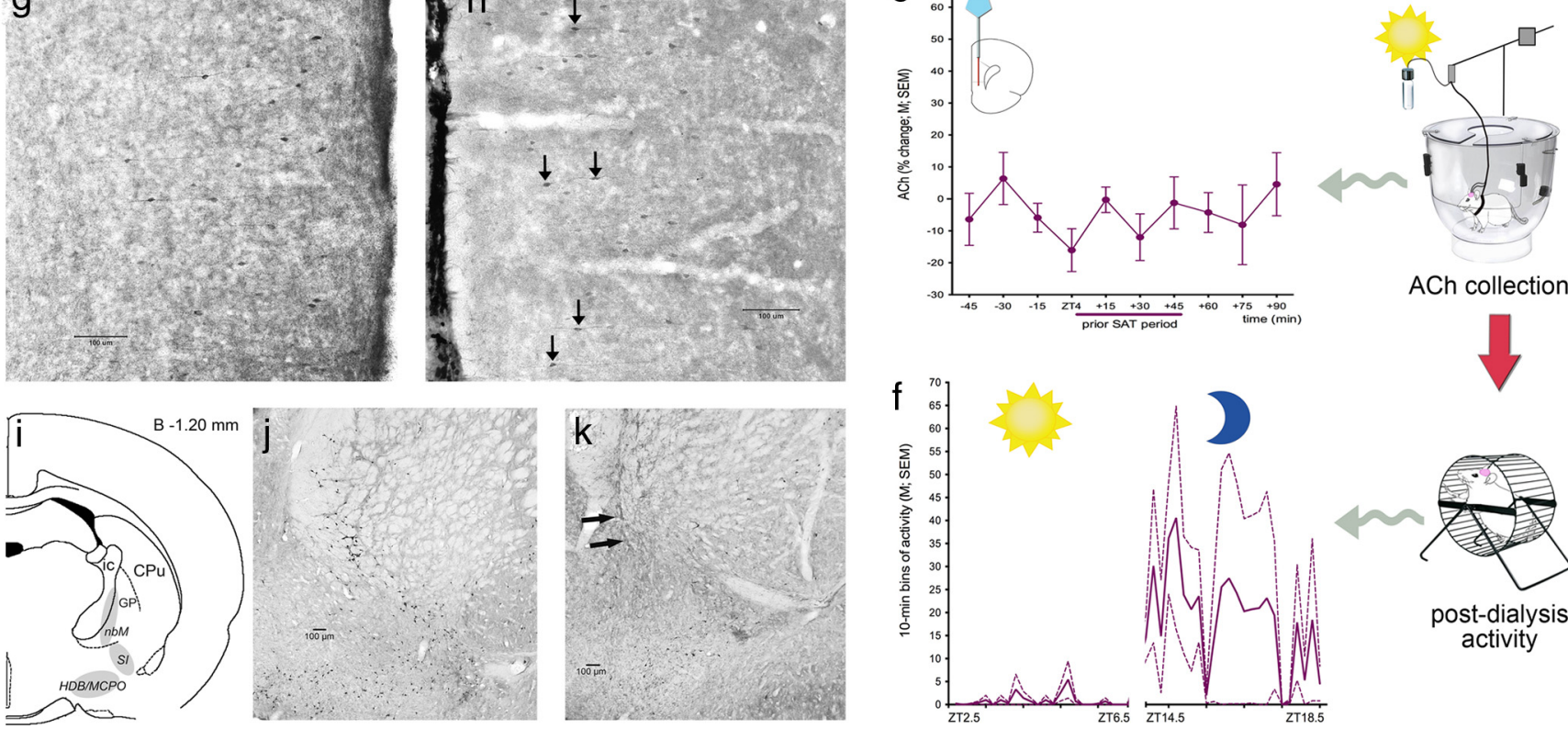

Figure 5. Cholinergic lesions impair SAT performance, abolish synchronized ACh release, and facilitate the reemergence of a nocturnal activity pattern. $\boldsymbol{a}$, Timeline and major experimental events (the wavy arrows linking schematics and data plots depict the origin of data across these events). Following $90 \mathrm{~d}$ of SAT practice at ZT4 and activity monitoring, bilateral infusions of the immunotoxin $192 \mathrm{lg}$ saporin into the basal forebrain $(n=7)$ removed $>80 \%$ of cortical cholinergic inputs $(\boldsymbol{g}-\boldsymbol{k}$; below). Following $50 \mathrm{~d}$ of postlesion SAT performance and activity monitoring, microdialysis guide cannula were implanted, followed by $10 \mathrm{~d}$ of SAT practice and then by transfer to the bowls and subsequent microdialysis and activity monitoring identical with the procedures in Figure $1 a$. $\boldsymbol{b}$, Before the lesions, SAT performance at ZT4 was associated with a diurnal activity pattern (wheels). c, SAT performance averaged over the last three sessions of the postcannulation $10 \mathrm{~d}$ performance period. SAT scores (abscissa) combine signal and nonsignal trial performance and are calculated over signal durations (see Materials and Methods) and range from 0 (random responding) to 1 (all response are hits or correct rejections). As expected and was repeatedly demonstrated, the lesions robustly and persistently impaired SAT performance, with SAT scores calculated over hits for the two shorter signal durations reaching below chance levels (see dashed line; post hoc multiple comparisons: ${ }^{*} p<0.05,{ }^{* *} p<0.001$ ). Error bars indicate SEM. $\boldsymbol{d}$, The final three SAT sessions were associated with a predominantly diurnal activity pattern, although to a lesser degree than observed in intact animals (Fig. $5 b$; see Results). $\boldsymbol{e}$, Residual ACh release in lesioned animals did not vary across collections that included the prior SAT practice period. $f$, Four days following the last SAT session, a completely nocturnal activity pattern had reemerged. This contrasts with a persistent diurnal activity pattern in intact animals at this point (Fig. 2b). $\boldsymbol{g}, \boldsymbol{h}, \mathrm{ChAT}$-immunostained sections of the medial prefrontal cortex of a control and a lesioned rat, respectively. Scale bars: $\boldsymbol{g}, \boldsymbol{h}, \boldsymbol{j}, \boldsymbol{k}, 100 \mu \mathrm{m}$. ChAT-positive puncta are distributed across all layers of the cortex $(\boldsymbol{g})$. Immunotoxin-induced removal of cholinergic inputs spared the bipolar ChAT-positive cortical interneurons that are mostly located in layers 2 and 3 (see arrows in $\boldsymbol{h}$ ). The postsynaptic action and, generally, the function of these interneurons are largely unclear (von Engelhardt et al., 2007). $\boldsymbol{i}$, Schematic location of the main cholinergic cell groups in the basal forebrain (shaded areas; CPu, caudate-putamen; GP, globus palidus; HDB/MCP0, horizontal nucleus of the diagonal band/magnocellular preoptic nucleus; $n b M$, nucleus basalis of Meynert; SI, substantia innominata). $j$, Distribution of ChAT-positive neurons in the basal forebrain in the nBM, SI, and HDB/MCPO (see i for orientation). $\boldsymbol{k}$, Infusions of the immunotoxin (see arrows for needle tract) removed the majority of $\mathrm{nbM}$ and $\mathrm{Si}$ cholinergic neurons, yielding a $>80 \%$ deafferentation of the cortex as indicated by measures of ChAT-positive fiber densities. 


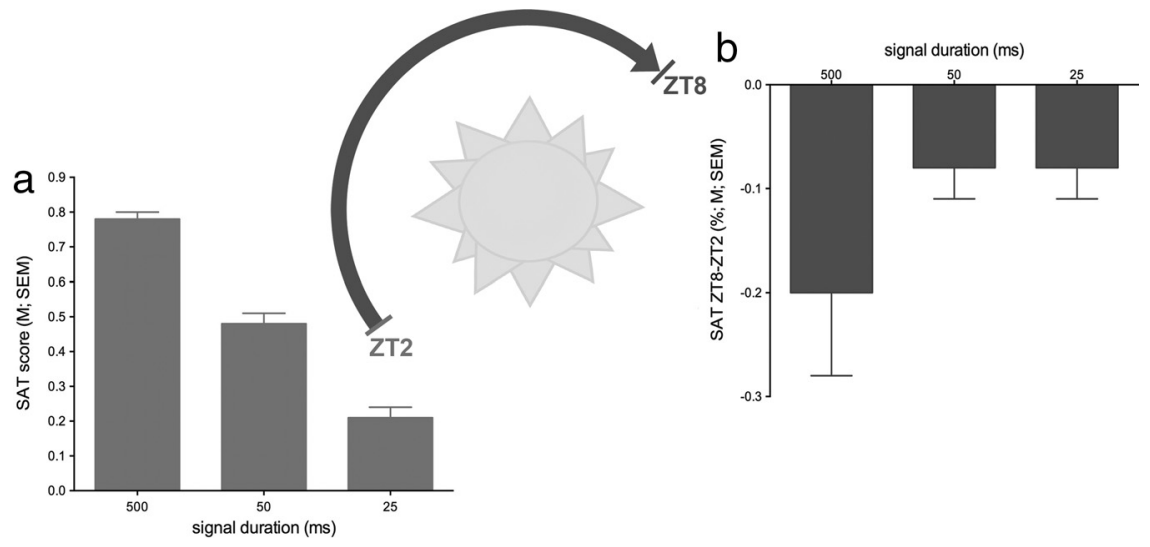

Figure 6. Impairments in SAT performance as a result of a phase delay of practice time by 6 h. $\boldsymbol{a}$, SAT scores calculated over signal durations averaged over the final three sessions of $>90 \mathrm{~d}$ of SAT practice at ZT2. SAT practice time was then moved to ZT8 $(n=8) . \boldsymbol{b}$, Impairments in SAT performance averaged over three sessions after the shift of practice time to ZT8 (see Results for ANOVA of the effects of shift). Performance remained impaired for this period following the shift of practice time, consistent with prior evidence indicating that weeks of practice are required to regain preshift baseline performance (Gritton et al., 2012). Error bars indicate SEM.

response rates produced by this operant schedule. After SAT practice was terminated, a nocturnal activity pattern resumed $8 \mathrm{~d}$ after the final SAT session and task period-synchronized cholinergic activation was no longer observed. (3) Food-restricted animals exhibited strong FAA and attenuated activity during the dark period (Hut et al., 2011). However, FAA was not associated with increases in prefrontal cholinergic neurotransmission. (4) Removal of cholinergic projections to the cortex robustly impaired SAT performance, attenuated performance-associated diurnality, and accelerated the return of nocturnality within $4 \mathrm{~d}$ after the last performance session. (5) Consistent with the hypothesis that task period-synchronized increases in cortical cholinergic activity benefits SAT performance, shifting the time of SAT practice within the light phase impaired performance.

The tonic component of cortical cholinergic neurotransmission modulates cortical circuitry to facilitate the attentional processing of stimuli (McKenna et al., 1989; Dalley et al., 2004; Disney et al., 2007; Herrero et al., 2008; Bauer et al., 2012). In the specific context of the SAT, tonic cholinergic activity upregulates the interactions between cue-evoked thalamic glutamatergic and a separate, phasic component of cholinergic neurotransmission, thereby increasing the probability of hits in attention tasks (Parikh et al., 2007, 2008; Howe et al., 2010; Hasselmo and Sarter, 2011). Compared with increases in cholinergic activity that are synchronized to the prior task practice time, reaching $60 \%$ over baseline, SAT performance-associated levels of ACh release are approximately twofold higher and, under conditions that require enhanced stimulus processing and distractor filtering, reach $180 \%$ over baseline (St. Peters et al., 2011a). Prior task period synchronized increases in cholinergic activity are hypothesized to contribute to the readiness of cortical circuitry to process thalamic inputs (Edeline et al., 1994). The additional increases in cholinergic activity that occur during actual task performance likely benefit distractor filtering (Gill et al., 2000) and motivated attentional performance over longer periods of time (Sarter et al., 2006). Thus, SAT task time-stamped increases in cholinergic activation benefit attentional performance if practiced precisely every $24 \mathrm{~h}$, as also indicated by the costs of shifts in performance time (below).

The increases in cholinergic activity were precisely synchronized to the prior SAT practice period. Even in animals main- tained under constant-light conditions (LL), and thus in the absence of a potent zeitgeber that could have assisted in timing the prior task period, the timing of the activation of the cholinergic system began one collection before task onset. Thus, SAT practice acts as a potent nonphotic zeitgeber. In animals that had practiced the SAT in the dark phase, beginning at ZT16, cholinergic activation coincided with prior task onset time but lasted twice the duration of prior task practice, perhaps reflecting the generally greater excitability of this neuronal system during the dark phase.

Our evidence indicates that the return of nocturnal activity was associated with the loss of task time-stamped cholinergic activity and that cholinergic lesions facilitate such a return of a nocturnal activity pattern. These findings suggests that timestamped increases in cholinergic neurotransmission are not just an expression of finely tuned clocks but in turn may also serve as a zeitgeber, to maintain the precise timing of prior task practice periods. Following $8 \mathrm{~d}$ without SAT practice, this bidirectional interaction between cholinergic activity and central and peripheral clocks was weakened, to the point that task period-synchronized ACh release was no longer observed.

The regulation and function of synchronized increases in cortical cholinergic activity may extend to basal forebrain cholinergic projections to the suprachiasmatic nucleus (SCN) (Erhardt et al., 2004; Hut and Van der Zee, 2011). It is not known whether SAT performance activates basal forebrain cholinergic projections to the SCN in parallel to the increases in cholinergic neurotransmission in the cortex. However, selective removal of SCN cholinergic afferents attenuates the diurnality-inducing effect of daily SAT practice at ZT4 (H. J. Gritton, A. M. Stasiak, M. Sarter, T. M. Lee, unpublished observations). We can therefore not rule out that diurnality associated with performing the SAT at ZT4 was maintained, at least in part, by activation of cholinergic inputs to the SCN, and that the rapid reemergence of nocturnal activity in lesioned animals was due, at least in part, to loss of cholinergic projections to the SCN (Bina et al., 1997).

Our results specify the type of behavior that was sufficient for elevating cortical ACh release at precisely the time of prior task practice. Neither performance of an operant schedule of reinforcement nor FAA was associated with such an effect. Furthermore, as spatial memory performance does not induce a diurnal activity pattern (Gritton et al., 2012) and does not depend on the cholinergic basal forebrain (Baxter et al., 1996; Dornan et al., 1996; Vuckovich et al., 2004), task practice-induced diurnality appears to result specifically from practicing an attentional task (van der Heijden et al., 2010) and the recruitment of underlying cortical cholinergic mechanisms.

Additional neuronal mechanisms associated with SAT performance may contribute to maintaining a diurnal activity pattern, particularly after removal of cholinergic projections, as indicated by the persistent diurnality in lesioned animals (Fig. 1d). However, the cholinergic lesion-induced rapid and complete return of a nocturnal activity after termination of SAT practice suggests that time-stamped activation of the basal cholinergic system is 
necessary for maintaining a diurnal activity pattern in the absence of daily SAT practice.

The neuronal mechanisms mediating the precisely synchronized increase in cholinergic neurotransmission, as well as the mechanisms mediating cholinergic entrainment remain speculative (de Prado et al., 2003). Concerning the former, evidence indicates that mesolimbic dopaminergic activity is strongly influenced by the SCN (Agostino et al., 2011). We previously demonstrated that activity in the nucleus accumbens is necessary and sufficient for elevating performance-associated increases in cortical cholinergic activity (Neigh-McCandless et al., 2002; Neigh et al., 2004; St. Peters et al., 2011a). Thus cholinergic activation may be timed via circuits involving mesolimbic dopaminergic neurons and/or via direct, hitherto-unknown mechanisms directly linking cholinergic cells to SCN output. Separate circuitry and local, including cortical, brain clocks (Kyriacou and Hastings, 2010) may control the timing of activation of neuronal systems mediating specific cognitive functions and more global behavioral processes, respectively [see also the discussion in the study by McGregor et al. (2011)].

The present evidence demonstrates the power and precision of the timing of neuronal mechanisms that are necessary for attentional performance. Our results from the final experiment are consistent with the hypothesis that attentional practice timestamped increases in cholinergic activity benefit attentional performance. However, time-stamped increases in cholinergic neurotransmission were assessed in a new environment to avoid contextual confounds, that is, the activating effects of being placed into the task chamber. Animals practicing at the same time and placed in the test chambers exhibit contextual and anticipatory increases in ACh release before task onset [Kozak et al. (2007), their Fig. 4]. Thus, while our results from the final experiment indicate the functionality of practicing attentiondemanding tasks at a fixed time every $24 \mathrm{~h}$ (Stroebel, 1967; Holloway and Wansley, 1973; Folkard et al., 1983; Gerstner and Yin, 2010), the relative contributions of, and interactions between, timing and contextual occasion setters, such as the taskrelated environment, to cholinergic activation and performance would need to be specified by complex experiments attempting to isolate these variables.

Finally, precise timing of attention-mediating neuronal circuitry demonstrated in the present experiments provides a neuronal mechanism underlying not only the benefits of fixed-time attentional performance but also accounting for the performance costs of variably timed performance (Schmidt et al., 2007) and, more dramatically, of performance shifted across light/dark phases (Fekete et al., 1985; Lustig and Meck, 2001; Reid et al., 2011). Evidence from humans indicating that the cognitive/attentional costs of circadian manipulations can be rescued in part by administering a cholinesterase inhibitor (Chuah and Chee, 2008) is consistent with the hypothesis that timed cholinergic activity is a mechanism that benefits fixed-time performance and also serves as a cognitive zeitgeber. Furthermore, major cognitive-affective symptoms of neuropsychiatric disorders have been associated with, or even attributed in part to, circadian dysregulation (Wulff and Joyce, 2011; Wulff et al., 2012). Such dysregulation may extend to the disruption of timed cholinergic activity, perhaps via circuitry including mesolimbic projections to the basal forebrain (Dzirasa et al., 2010; Mukherjee et al., 2010).

In conclusion, these experiments demonstrated that fixedtime, daily practice of a task requiring cortical cholinergic activity generates a precisely practice time-stamped activation of this neuronal system. Practice time-synchronized cholinergic activa- tion is expressed independently of the task environment and the presence of photic zeitgebers. Time-stamped cholinergic activation benefits fixed-time performance and, if practiced during the light phase, contributes to a diurnal activity pattern. Numerous behavioral and cognitive routines may benefit from timestamped activation of their underlying neuronal mechanisms. Circadian dysregulation, caused either by phase shifts or associated with neuropsychiatric disorders, can therefore be hypothesized to cause impairments in attentional performance as a result of ill timed or absent activation of the cortical cholinergic input system.

\section{References}

Agostino PV, Golombek DA, Meck WH (2011) Unwinding the molecular basis of interval and circadian timing. Front Integr Neurosci 5:64.

Arnold HM, Burk JA, Hodgson EM, Sarter M, Bruno JP (2002) Differential cortical acetylcholine release in rats performing a sustained attention task versus behavioral control tasks that do not explicitly tax attention. Neuroscience 114:451-460

Atalayer D, Rowland NE (2011) Comparison of voluntary and foraging running wheel activity on food demand in mice. Physiol Behav 102:22-29.

Bauer M, Kluge C, Bach D, Bradbury D, Heinze HJ, Dolan RJ, Driver J (2012) Cholinergic enhancement of visual attention and neural oscillations in the human brain. Curr Biol 22:397-402.

Baxter MG, Bucci DJ, Sobel TJ, Williams MJ, Gorman LK, Gallagher M (1996) Intact spatial learning following lesions of basal forebrain cholinergic neurons. Neuroreport 7:1417-1420.

Bergua V, Fabrigoule C, Barberger-Gateau P, Dartigues JF, Swendsen J, Bouisson J (2006) Preferences for routines in older people: associations with cognitive and psychological vulnerability. Int J Geriatr Psychiatry 21:990-998.

Bina KG, Rusak B, Semba K (1997) Sources of p75-nerve growth factor receptor-like immunoreactivity in the rat suprachiasmatic nucleus. Neuroscience 77:461-472.

Boulos Z, Rosenwasser AM, Terman M (1980) Feeding schedules and the circadian organization of behavior in the rat. Behav Brain Res 1:39-65.

Campbell BA, Lynch GS (1968) Influence of hunger and thirst on the relationship between spontaneous activity and body temperature. J Comp Physiol Psychol 65:492-498.

Chee MW, Chuah LY, Venkatraman V, Chan WY, Philip P, Dinges DF (2006) Functional imaging of working memory following normal sleep and after 24 and $35 \mathrm{~h}$ of sleep deprivation: correlations of fronto-parietal activation with performance. Neuroimage 31:419-428.

Chuah LY, Chee MW (2008) Cholinergic augmentation modulates visual task performance in sleep-deprived young adults. J Neurosci 28:11369-11377.

Collier G, Hirsch E (1971) Reinforcing properties of spontaneous activity in the rat. J Comp Physiol Psychol 77:155-160.

Daan S (2000) Learning and circadian behavior. J Biol Rhythms 15:296-299.

Daan S, Pittendrigh CS (1976) A functional analysis of circadian pacemakers in nocturnal rodents. J Comp Physiol A Neuroethol Sens Neural Behav Physiol 106:267-290.

Dalley JW, McGaughy J, O’Connell MT, Cardinal RN, Levita L, Robbins TW (2001) Distinct changes in cortical acetylcholine and noradrenaline efflux during contingent and noncontingent performance of a visual attentional task. J Neurosci 21:4908-4914.

Dalley JW, Theobald DE, Bouger P, Chudasama Y, Cardinal RN, Robbins TW (2004) Cortical cholinergic function and deficits in visual attentional performance in rats following 192 IgG-saporin-induced lesions of the medial prefrontal cortex. Cereb Cortex 14:922-932.

Demeter E, Sarter M, Lustig C (2008) Rats and humans paying attention: cross-species task development for translational research. Neuropsychology 22:787-799.

de Prado BM, Reiter RJ, Mora F (2003) Perfusion of melatonin into the prefrontal cortex disrupts the circadian rhythm of acetylcholine but not of locomotor activity. J Pineal Res 35:283-287.

Disney AA, Aoki C, Hawken MJ (2007) Gain modulation by nicotine in macaque v1. Neuron 56:701-713.

Dornan WA, McCampbell AR, Tinkler GP, Hickman LJ, Bannon AW, Decker MW, Gunther KL (1996) Comparison of site-specific injections into the 
basal forebrain on water maze and radial arm maze performance in the male rat after immunolesioning with 192 IgG saporin. Behav Brain Res 82:93-101.

Dzirasa K, Coque L, Sidor MM, Kumar S, Dancy EA, Takahashi JS, McClung CA, Nicolelis MA (2010) Lithium ameliorates nucleus accumbens phase-signaling dysfunction in a genetic mouse model of mania. J Neurosci 30:16314-16323.

Echevarria DJ, Brewer A, Burk JA, Brown SN, Manuzon H, Robinson JK (2005) Construct validity of an operant signal detection task for rats. Behav Brain Res 157:283-290.

Edeline JM, Maho C, Hars B, Hennevin E (1994) Non-awaking basal forebrain stimulation enhances auditory cortex responsiveness during slowwave sleep. Brain Res 636:333-337.

Erhardt C, Galani R, Jeltsch H, Cassel JC, Klosen P, Menet JS, Pévet P, Challet E (2004) Modulation of photic resetting in rats by lesions of projections to the suprachiasmatic nuclei expressing p75 neurotrophin receptor. Eur J Neurosci 19:1773-1788.

Fekete M, van Ree JM, Niesink RJ, de Wied D (1985) Disrupting circadian rhythms in rats induces retrograde amnesia. Physiol Behav 34:883-887.

Folkard S, Wever RA, Wildgruber CM (1983) Multi-oscillatory control of circadian rhythms in human performance. Nature 305:223-226.

Frey PW, Colliver JA (1973) Sensitivity and responsivity measures for discrimination learning. Learn Motiv 4:327-342.

Gerstner JR, Yin JC (2010) Circadian rhythms and memory formation. Nat Rev Neurosci 11:577-588.

Gill TM, Sarter M, Givens B (2000) Sustained visual attention performanceassociated prefrontal neuronal activity: evidence for cholinergic modulation. J Neurosci 20:4745-4757.

Greenwald AG, Gonzalez R, Harris RJ, Guthrie D (1996) Effect sizes and $p$ values: what should be reported and what should be replicated? Psychophysiology 33:175-183.

Gritton HJ, Sutton BC, Martinez V, Sarter M, Lee TM (2009) Interactions between cognition and circadian rhythms: attentional demands modify circadian entrainment. Behav Neurosci 123:937-948.

Gritton HJ, Kantorowski A, Sarter M, Lee TM (2012) Bidirectional interactions between circadian entrainment and cognitive performance. Learn Mem 19:126-141.

Hasselmo ME, Sarter M (2011) Modes and models of forebrain cholinergic neuromodulation of cognition. Neuropsychopharmacology 36:52-73.

Herrero JL, Roberts MJ, Delicato LS, Gieselmann MA, Dayan P, Thiele A (2008) Acetylcholine contributes through muscarinic receptors to attentional modulation in V1. Nature 454:1110-1114.

Holloway FA, Wansley RA (1973) Multiple retention deficits at periodic intervals after active and passive avoidance learning. Behav Biol 9:1-14.

Howe WM, Ji J, Parikh V, Williams S, Mocaër E, Trocmé-Thibierge C, Sarter M (2010) Enhancement of attentional performance by selective stimulation of alpha4beta $2^{*}$ nAChRs: underlying cholinergic mechanisms. Neuropsychopharmacology 35:1391-1401.

Hut RA, Van der Zee EA (2011) The cholinergic system, circadian rhythmicity, and time memory. Behav Brain Res 221:466-480.

Hut RA, Pilorz V, Boerema AS, Strijkstra AM, Daan S (2011) Working for food shifts nocturnal mouse activity into the day. PLoS One 6:e17527.

Jiménez-Capdeville ME, Dykes RW (1996) Changes in cortical acetylcholine release in the rat during day and night: differences between motor and sensory areas. Neuroscience 71:567-579.

Kametani H, Kawamura H (1991) Circadian rhythm of cortical acetylcholine release as measured by in vivo microdialysis in freely moving rats. Neurosci Lett 132:263-266.

Kozak R, Martinez V, Young D, Brown H, Bruno JP, Sarter M (2007) Toward a neuro-cognitive animal model of the cognitive symptoms of schizophrenia: disruption of cortical cholinergic neurotransmission following repeated amphetamine exposure in attentional task-performing, but not non-performing, rats. Neuropsychopharmacology 32:2074-2086.

Kyriacou CP, Hastings MH (2010) Circadian clocks: genes, sleep, and cognition. Trends Cogn Sci 14:259-267.

Lustig C, Meck WH (2001) Paying attention to time as one gets older. Psychol Sci 12:478-484.

Matchock RL, Mordkoff JT (2009) Chronotype and time-of-day influences on the alerting, orienting, and executive components of attention. Exp Brain Res 192:189-198.

McGaughy J, Sarter M (1995) Behavioral vigilance in rats: task validation and effects of age, amphetamine, and benzodiazepine receptor ligands. Psychopharmacology (Berl) 117:340-357.

McGaughy J, Sarter M (1998) Sustained attention performance in rats with intracortical infusions of $192 \mathrm{IgG}$-saporin-induced cortical cholinergic deafferentation: effects of physostigmine and FG 7142. Behav Neurosci 112:1519-1525

McGaughy J, Kaiser T, Sarter M (1996) Behavioral vigilance following infusions of 192 IgG-saporin into the basal forebrain: selectivity of the behavioral impairment and relation to cortical AChE-positive fiber density. Behav Neurosci 110:247-265.

McGaughy J, Everitt BJ, Robbins TW, Sarter M (2000) The role of cortical cholinergic afferent projections in cognition: impact of new selective immunotoxins. Behav Brain Res 115:251-263.

McGregor R, Wu MF, Barber G, Ramanathan L, Siegel JM (2011) Highly specific role of hypocretin (orexin) neurons: differential activation as a function of diurnal phase, operant reinforcement versus operant avoidance and light level. J Neurosci 31:15455-15467.

McKenna TM, Ashe JH, Weinberger NM (1989) Cholinergic modulation of frequency receptive fields in auditory cortex. I. Frequency-specific effects of muscarinic agonists. Synapse 4:30-43.

Meck WH (1991) Modality-specific circadian rhythmicities influence mechanisms of attention and memory for interval timing. Learn Motiv 22:153-179.

Mendoza J, Graff C, Dardente H, Pevet P, Challet E (2005) Feeding cues alter clock gene oscillations and photic responses in the suprachiasmatic nuclei of mice exposed to a light/dark cycle. J Neurosci 25:1514-1522.

Mistlberger RE (1994) Circadian food-anticipatory activity: formal models and physiological mechanisms. Neurosci Biobehav Rev 18:171-195.

Mistlberger RE, Kent BA, Landry GJ (2009) Phenotyping food entrainment: motion sensors and telemetry are equivalent. J Biol Rhythms 24:95-98.

Mollicone DJ, Van Dongen HP, Rogers NL, Banks S, Dinges DF (2010) Time of day effects on neurobehavioral performance during chronic sleep restriction. Aviat Space Environ Med 81:735-744.

Mukherjee S, Coque L, Cao JL, Kumar J, Chakravarty S, Asaithamby A, Graham A, Gordon E, Enwright JF 3rd, DiLeone RJ, Birnbaum SG, Cooper DC, McClung CA (2010) Knockdown of Clock in the ventral tegmental area through RNA interference results in a mixed state of mania and depression-like behavior. Biol Psychiat 68:503-511.

Neigh GN, Arnold HM, Rabenstein RL, Sarter M, Bruno JP (2004) Neuronal activity in the nucleus accumbens is necessary for performance-related increases in cortical acetylcholine release. Neuroscience 123:635-645.

Neigh-McCandless G, Kravitz BA, Sarter M, Bruno JP (2002) Stimulation of cortical acetylcholine release following blockade of ionotropic glutamate receptors in nucleus accumbens. Eur J Neurosci 16:1259-1266.

Paolone G, Howe WM, Gopalakrishnan M, Decker MW, Sarter M (2010) Regulation and function of the tonic component of cortical acetylcholine release. In: Monitoring molecules in neuroscience (Westerink B, Clinckers R, Smolders S, Sarre S, Michotte Y, eds), pp 363-365. Brussels: Vrije Universiteit Brussels.

Parikh V, Kozak R, Martinez V, Sarter M (2007) Prefrontal acetylcholine release controls cue detection on multiple timescales. Neuron 56:141-154.

Parikh V, Man K, Decker MW, Sarter M (2008) Glutamatergic contributions to nicotinic acetylcholine receptor agonist-evoked cholinergic transients in the prefrontal cortex. J Neurosci 28:3769-3780.

Parikh V, Ji J, Decker MW, Sarter M (2010) Prefrontal $\beta 2$ subunitcontaining and $\alpha 7$ nicotinic acetylcholine receptors differentially control glutamatergic and cholinergic signaling. J Neurosci 30:3518-3530.

Pittendrigh CS, Daan S (1976) A functional analysis of circadian pacemakers in nocturnal rodents. J Comp Physiol A Neuroethol Sens Neural Behav Physiol 106:291-331.

Posner MI, Snyder CR, Davidson BJ (1980) Attention and the detection of signals. J Exp Psychol 109:160-174.

Reid KJ, McGee-Koch LL, Zee PC (2011) Cognition in circadian rhythm sleep disorders. Prog Brain Res 190:3-20.

Rosenwasser AM, Pellowski MW, Hendley ED (1996) Circadian timekeeping in hyperactive and hypertensive inbred rat strains. Am J Physiol 271:R787-R796.

Sarter M, Parikh V (2005) Choline transporters, cholinergic transmission and cognition. Nat Rev Neurosci 6:48-56.

Sarter M, Gehring WJ, Kozak R (2006) More attention must be paid: the neurobiology of attentional effort. Brain Res Rev 51:145-160. 
Schmidt C, Collette F, Cajochen C, Peigneux P (2007) A time to think: circadian rhythms in human cognition. Cogn Neuropsychol 24:755-789.

Sherwin CM (1998) Voluntary wheel running: a review and novel interpretation. Anim Behav 56:11-27.

Stephan FK (2002) The "other" circadian system: food as a zeitgeber. J Biol Rhythms 17:284-292.

St. Peters M, Demeter E, Lustig C, Bruno JP, Sarter M (2011a) Enhanced control of attention by stimulating mesolimbic-corticopetal cholinergic circuitry. J Neurosci 31:9760-9771.

St. Peters M, Cherian AK, Bradshaw M, Sarter M (2011b) Sustained attention in mice: expanding the translational utility of the SAT by incorporating the Michigan Controlled Access Response Port (MICARP). Behav Brain Res 225:574-583.

Stroebel CF (1967) Behavioral aspects of circadian rhythms. In: Comparative psychopathology: animal and human (Zubin J, Hunt HF, eds), pp 158-172. New York: Grune and Stratton.
Treichler FR, Hall JF (1962) The relationship between deprivation weight loss and several measures of activity. J Comp Physiol Psychol 55:346-349.

van der Heijden KB, de Sonneville LM, Althaus M (2010) Time-of-day effects on cognition in preadolescents: a TRAILS study. Chronobiol Int 27:1870-1894.

von Engelhardt J, Eliava M, Meyer AH, Rozov A, Monyer H (2007) Functional characterization of intrinsic cholinergic interneurons in the cortex. J Neurosci 27:5633-5642.

Vuckovich JA, Semel ME, Baxter MG (2004) Extensive lesions of cholinergic basal forebrain neurons do not impair spatial working memory. Learn Mem 11:87-94.

Wulff K, Joyce E (2011) Circadian rhythms and cognition in schizophrenia. Br J Psychiatry 198:250-252.

Wulff K, Dijk DJ, Middleton B, Foster RG, Joyce EM (2012) Sleep and circadian rhythm disruption in schizophrenia. Br J Psychiatry 200: $308-316$. 